QUARTERLY OF APPLIED MATHEMATICS

VOLUME LXIII, NUMBER 4

DECEMBER 2005, PAGES 645-672

S $0033-569 X(05) 00983-2$

Article electronically published on September 22, 2005

\title{
MINIMUM FREE ENERGY IN LINEAR THERMOELECTROMAGNETISM
}

\author{
BY \\ GIOVAMBATTISTA AMENDOLA (Dipartimento di Matematica Applicata "U.Dini", Facoltà di \\ Ingegneria, via Diotisalvi 2, 56126-Pisa, Italy) \\ AND
}

ADELE MANES (Dipartimento di Matematica "L.Tonelli", via F.Buonarroti 2, 56127-Pisa, Italy)

\begin{abstract}
A closed expression is given for the minimum free energy of a linear thermoelectromagnetic conductor, whose constitutive equations relative to the electric current density and to the heat flux have memory effects. This expression, derived in the frequency domain, is related to the maximum recoverable work, which can be obtained from a given state of the material. Another equivalent expression of the minimum free energy is deduced; it allows us to give explicit formulae for the case of a discrete spectrum model.
\end{abstract}

1. Introduction. The investigation of the problem of finding explicit forms of the minimum free energy of a viscoelastic solid has been considered by many authors because of the importance of the maximum recoverable work, to which the minimum free energy is related (see, for example, [1, 2, 3, 5, 8, 9, 12, 16]). Two of these works [3, 5] are particularly interesting for the method used in studying such a problem; therefore, we shall refer to these papers, since we shall use their procedure for finding the expression of the minimum free energy of a thermoelectromagnetic conductor.

Also in electromagnetism this problem has been studied in recent articles; in particular, in [6] the minimum free energy has been evaluated for a rigid dielectric material with linear memory under isothermal conditions, the material being characterized by memory effects for the electric displacement and the magnetic induction. In [14 the same problem has been considered for an electromagnetic conductor whose behaviour is similar to that of the ionosphere, for which the hereditary theory provides a local functional relation between the electric current density and the electric field [4, 13.

Received December 1, 2004.

2000 Mathematics Subject Classification. Primary 78A25, 74A15.

Key words and phrases. Thermoelectromagnetism, fading memory, free energy.

This work was performed under the support of C.N.R. and M.I.U.R.

(C)2005 Brown University 
In this paper we are concerned with the presence of thermal effects in electromagnetism. They have been considered by Coleman and Dill in [19, 20, where the thermodynamic restrictions on the constitutive equations have been derived, too (see also [7). In particular, these effects have also been studied in [15, where the linear theory of the thermodynamics of a homogeneous conductor is considered, by assuming that the material is characterized by two linearized constitutive equations, which exhibit longterm memory, for the electric current density and the heat flux. Both of these equations have the linearized form introduced in [17] for the heat flux to remove the paradox of the classic theory about the instantaneous propagation of heat [18. The linearized theory developed in 15 yields the introduction of a maximal free energy, which allows us to obtain a domain of dependence; moreover, a theorem of uniqueness, existence and asymptotic stability is proved. In this work we address ourselves to the problem of finding the expression of the minimum free energy of the thermoelectromagnetic conductor, already examined in [15]. An explicit formula is derived by starting from the formulation of the maximum recoverable work and using the notion of minimal state, that is, the state which considers equivalent the histories which yield the same response of the material [16, 22. The technique used to obtain such a formula starts from the study of two Wiener-Hopf integral equations of the first kind, whose solutions are derived by considering the thermodynamic properties of the integral kernels and some theorems on the factorization of the same kernels.

In Sec. 2 the constitutive equations are introduced and the thermodynamic restrictions on them are also recalled [15. Then, in Sec. 3, some useful properties of the two integral kernels of the functionals, which characterize the electric current density and the heat flux, are derived; moreover, we introduce the notions of state and process, which describe the behaviour of the material 21, 22. The position of the problem and the definition of the continuation of histories with a given process are considered in Sec. 4, where the definition of equivalent histories is also given. Then, in Sec. 5, we introduce the thermoelectromagnetic work done on a process starting from a given state, and we derive some expressions of this work by distinguishing different initial states. In Sec. 6 we give a new definition of equivalent histories by means of the work based on the boundedness of the same work; we show that the two definitions of equivalence are equivalent. Then, in Sec. 7, we consider the maximum recoverable work, which is related to the minimum free energy, of which an explicit form is obtained. After deriving, in Sec. 8, a different but equivalent formulation for the minimum free energy, in the last section we consider the particular case of a discrete spectrum model material response, for which explicit formulae are derived.

2. Preliminaries. Let $\mathcal{B}$ be an electromagnetic solid, which occupies a region $\Omega$ of the three-dimensional Euclidean space $\mathbf{R}^{3}$. We suppose that $\Omega$ is a bounded and regular domain with a smooth boundary.

Within the linear theory of thermoelectromagnetism, $\mathcal{B}$ is considered as a homogeneous and isotropic conductor, characterized, in particular, by the presence of memory effects for the electric current density $\mathbf{J}$ and the heat flux $\mathbf{q}$. Denoting by $\mathbf{D}$ the electric 
displacement, B the magnetic induction, $\vartheta$ the temperature relative to the absolute reference temperature $\Theta_{0}$, uniform in $\Omega, \mathbf{E}$ and $\mathbf{H}$ the electric and magnetic fields and $h$ the rate at which heat is absorbed per unit volume, we assume the following constitutive equations:

$$
\begin{aligned}
\mathbf{D}(\mathbf{x}, t) & =\varepsilon \mathbf{E}(\mathbf{x}, t)+\vartheta(\mathbf{x}, t) \mathbf{a}, \quad \mathbf{B}(\mathbf{x}, t)=\mu \mathbf{H}(\mathbf{x}, t) \\
\mathbf{J}(\mathbf{x}, t) & =\int_{0}^{+\infty} \alpha(s) \mathbf{E}(\mathbf{x}, t-s) d s, \quad \mathbf{q}(\mathbf{x}, t)=-\int_{0}^{+\infty} k(s) \mathbf{g}(\mathbf{x}, t-s) d s \\
h(\mathbf{x}, t) & =c \dot{\vartheta}(\mathbf{x}, t)+\Theta_{0}\left[\varepsilon^{-1} \mathbf{A}_{1} \cdot \dot{\mathbf{D}}(\mathbf{x}, t)+\mu^{-1} \mathbf{A}_{2} \cdot \dot{\mathbf{B}}(\mathbf{x}, t)\right] .
\end{aligned}
$$

Here the time $t \in \mathbf{R}^{+}, \mathbf{a}, \mathbf{A}_{1}$ and $\mathbf{A}_{2}$ are three constant vectors, the positive coefficients $\varepsilon, \mu$ and $c$ are the dielectric constant, the permeability and the specific heat. Moreover, the local functionals, which express $\mathbf{J}$ and $\mathbf{q}$, depend upon the histories of the electric field, $\mathbf{E}:(-\infty, t] \times \Omega \rightarrow \mathbf{R}^{3}$, and of the temperature gradient, $\mathbf{g}=\nabla \vartheta:(-\infty, t] \times \Omega \rightarrow \mathbf{R}^{3}$, up to time $t$.

All these functions depend upon the position $\mathbf{x} \in \Omega$, but henceforth it will be understood since the statements are relative to any fixed $\mathbf{x} \in \Omega$. In (2.2) the two kernels $\alpha: \mathbf{R}^{+} \rightarrow \mathbf{R}$ and $k: \mathbf{R}^{+} \rightarrow \mathbf{R}$ are the electric and the thermal conductivities, respectively; we suppose that these functions belong to $H^{1}\left(\mathbf{R}^{+}\right)$.

In [15] we have observed that the assumed constitutive equations allow us to consider $\mathcal{B}$ as a simple material [20, 21] and we have derived the restrictions imposed on them by the laws of thermodynamics, which state [10, 11] that for any cyclic process the equality

$$
\oint[h(t)+\mathbf{E}(t) \cdot \dot{\mathbf{D}}(t)+\mathbf{H}(t) \cdot \dot{\mathbf{B}}(t)+\mathbf{E}(t) \cdot \mathbf{J}(t)] d t=0
$$

holds as well as the inequality

$$
\oint\left\{\left[\Theta_{0}+\vartheta(t)\right]^{-1} h(t)+\left[\Theta_{0}+\vartheta(t)\right]^{-2} \mathbf{q}(t) \cdot \mathbf{g}(t)\right\} d t \leq 0,
$$

where the equality sign is relative only to reversible processes and $h$ in our case is expressed by $(2.3)$.

To deduce the required restrictions, it is necessary to use the approximate expression of (2.5) given by 9

$$
\Theta_{0}^{-2} \oint\left\{h(t)\left[\Theta_{0}-\vartheta(t)\right]+\mathbf{q}(t) \cdot \mathbf{g}(t)\right\} d t \leq 0 ;
$$

hence, using (2.4) we have

$$
\begin{aligned}
\Theta_{0}^{-2} \oint\{h(t) \vartheta(t)+ & \Theta_{0}[\mathbf{E}(t) \cdot \dot{\mathbf{D}}(t)+\mathbf{H}(t) \cdot \dot{\mathbf{B}}(t) \\
& +\mathbf{E}(t) \cdot \mathbf{J}(t)]-\mathbf{q}(t) \cdot \mathbf{g}(t)\} d t \geq 0 .
\end{aligned}
$$

Upon an integration on cycles, substituting (2.3) with (2.1), the inequality (2.7) gives a relation from which the arbitrarinesses of $\vartheta, \mathbf{E}$ and $\mathbf{g}$ and of $\vartheta$ with respect to $\dot{\mathbf{E}}$ and $\dot{\mathbf{H}}$ yield the thermodynamic restrictions 15 .

$$
\mathbf{A}_{1}=\mathbf{a}, \quad \mathbf{A}_{2}=\mathbf{0}
$$


together with two inequalities, which, as we have shown in [15], reduce to

$$
\int_{0}^{+\infty} \alpha(s) \cos (\omega s) d s>0, \quad \int_{0}^{+\infty} k(s) \cos (\omega s) d s>0 \quad \forall \omega \neq 0 .
$$

Thus, we observe that the expression (2.3), due to (2.1) and (2.8), becomes

$$
h(\mathbf{x}, t)=\left(c+\Theta_{0} \varepsilon^{-1} \mathbf{a}^{2}\right) \dot{\vartheta}(\mathbf{x}, t)+\Theta_{0} \mathbf{a} \cdot \dot{\mathbf{E}}(\mathbf{x}, t) ;
$$

moreover, substituting this relation and taking account of (2.1) allow us to write (2.7) as follows:

$$
\begin{aligned}
\oint\left[\frac{c}{\Theta_{0}} \dot{\vartheta}(t) \vartheta(t)\right. & +\frac{1}{\varepsilon} \dot{\mathbf{D}}(t) \cdot \mathbf{D}(t)+\frac{1}{\mu} \dot{\mathbf{B}}(t) \cdot \mathbf{B}(t) \\
& \left.+\mathbf{J}(t) \cdot \mathbf{E}(t)-\frac{1}{\Theta_{0}} \mathbf{q}(t) \cdot \mathbf{g}(t)\right] d t \geq 0,
\end{aligned}
$$

which must hold for any cyclic process.

We remember that the field equations in thermoelectromagnetism are Maxwell's equations together with the energy equation, which relates $h$ to $\mathbf{q}$, i.e. $h(\mathbf{x}, t)=-\nabla \cdot \mathbf{q}(\mathbf{x}, t)+$ $r(\mathbf{x}, t)$, where $r$ denotes the heat sources and $h$ can be eliminated by using the expression (2.10) in terms of $\dot{\vartheta}$ and $\dot{\mathbf{E}}$.

3. Some properties of the two kernels $\alpha$ and $k$. Some useful results can be derived by considering the two kernels $\alpha$ and $k$ which appear in (2.2).

To do this, we first note that any history can be considered as the set of the present value and of the past history; therefore, we shall identify, in particular, the history of the electric field $\mathbf{E}:(-\infty, t] \rightarrow \mathbf{R}^{3}$ with the couple $\left(\mathbf{E}(t), \mathbf{E}^{t}\right)$, where the past history is denoted by $\mathbf{E}^{t}(s)=\mathbf{E}(t-s) \forall s \in \mathbf{R}^{++}$.

We observe that the present value of the electric field must be considered not only for the history of this field but also because it appears in the constitutive equation $(2.1)_{1}$ of the electric displacement. On the contrary, the present value of the temperature gradient hasn't an equivalent role in the constitutive equations; therefore, only its past history $\mathbf{g}^{t}(s)=\mathbf{g}(t-s) \forall s \in \mathbf{R}^{++}$assumes a particular importance, as we shall see in the definition of the state of the material, too.

Now, let us recall the definition of the static continuation of any history; if the two histories $\left(\mathbf{E}(t), \mathbf{E}^{t}\right)$ and $\left(\mathbf{g}(t), \mathbf{g}^{t}\right)$ are considered, their static continuations with duration $a \in \mathbf{R}^{+}$are defined by

$$
\mathbf{E}^{t_{(a)}}=\left\{\begin{array}{ll}
\mathbf{E}^{t}(s-a), & s>a, \\
\mathbf{E}(t), & s \in[0, a],
\end{array} \mathbf{g}^{t_{(a)}}= \begin{cases}\mathbf{g}^{t}(s-a), & s>a, \\
\mathbf{g}(t), & s \in[0, a] .\end{cases}\right.
$$

Introducing the electric conductivity $\nu^{(\alpha)}$ and the thermal one $\nu^{(k)}$ by means of

$$
\nu^{(\alpha)}(t)=\int_{0}^{t} \alpha(\xi) d \xi, \quad \nu^{(k)}(t)=\int_{0}^{t} k(\xi) d \xi
$$


the current density and the heat flux yielded with the static continuations (3.1) are given by

$$
\begin{aligned}
& \mathbf{J}(t+a)=\nu^{(\alpha)}(a) \mathbf{E}(t)+\int_{0}^{+\infty} \alpha(a+\xi) \mathbf{E}^{t}(\xi) d \xi, \\
& \mathbf{q}(t+a)=-\nu^{(k)}(a) \mathbf{g}(t)-\int_{0}^{+\infty} k(a+\xi) \mathbf{g}^{t}(\xi) d \xi,
\end{aligned}
$$

where we note that the presence of $\mathbf{g}(t)$, as well as of $\mathbf{E}(t)$, is due, obviously, only to the static continuations of these values.

Thus, we introduce these spaces:

$$
\begin{array}{lll}
\Gamma_{\alpha}= & \left\{\mathbf{E}^{t}:(0,+\infty) \rightarrow \mathbf{R}^{3} ;\left|\int_{0}^{+\infty} \alpha(s+\tau) \mathbf{E}^{t}(s) d s\right|<+\infty \quad \forall \tau \geq 0\right\}, \\
\Gamma_{k}=\quad & \left\{\mathbf{g}^{t}:(0,+\infty) \rightarrow \mathbf{R}^{3} ;\left|\int_{0}^{+\infty} k(s+\tau) \mathbf{g}^{t}(s) d s\right|<+\infty \quad \forall \tau \geq 0\right\} .
\end{array}
$$

To derive other results we must introduce the Fourier transform $f_{F}$ of any function $f \in L^{1}(\mathbf{R}) \cap L^{2}(\mathbf{R})$; it is given by

$$
f_{F}(\omega)=\int_{-\infty}^{+\infty} f(\xi) e^{-i \omega \xi} d \xi=f_{+}(\omega)+f_{-}(\omega)
$$

where we have put

$$
f_{+}(\omega)=\int_{0}^{+\infty} f(\xi) e^{-i \omega \xi} d \xi, \quad f_{-}(\omega)=\int_{-\infty}^{0} f(\xi) e^{-i \omega \xi} d \xi
$$

When the functions are defined on $\mathbf{R}^{+}$we can identify them with their causal extensions to $(-\infty, 0)$, where they vanish identically, and (3.5) and (3.6) give

$$
f_{F}(\omega)=f_{+}(\omega)=f_{c}(\omega)-i f_{s}(\omega),
$$

where

$$
f_{c}(\omega)=\int_{0}^{+\infty} f(\xi) \cos (\omega \xi) d \xi, \quad f_{s}(\omega)=\int_{0}^{+\infty} f(\xi) \sin (\omega \xi) d \xi
$$

denote the Fourier cosine and sine transforms.

Finally, if the extension of any function defined on $\mathbf{R}^{+}$is made with an even function we have $f(\xi)=f(-\xi), \xi<0$, and $f_{F}(\omega)=2 f_{c}(\omega)$, while if it is made with an odd function we have $f(\xi)=-f(-\xi), \xi<0$, and $f_{F}(\omega)=-2 i f_{s}(\omega)$.

The thermodynamic restrictions (2.9), by virtue of $(3.8)_{1}$, become

$$
\alpha_{c}(\omega)>0, \quad k_{c}(\omega)>0 \quad \forall \omega \in \mathbf{R},
$$

under the hypotheses that $\alpha_{c}(0)>0$ and $k_{c}(0)>0$.

Thus, in particular, taking into account (3.2), we suppose that the following quantities are finite and positive, that is,

$$
\nu_{\infty}^{(\alpha)}=\int_{0}^{+\infty} \alpha(\xi) d \xi=\alpha_{c}(0)>0, \quad \nu_{\infty}^{(k)}=\int_{0}^{+\infty} k(\xi) d \xi=k_{c}(0)>0
$$


from which it follows that constant histories $\mathbf{E}^{t}=\mathbf{E}^{\dagger}$, defined as $\mathbf{E}^{t}(s)=\mathbf{E} \forall s \in \mathbf{R}^{+}$, and $\mathbf{g}^{t}=\mathbf{g}^{\dagger}$, given by $\mathbf{g}^{t}(s)=\mathbf{g} \forall s \in \mathbf{R}^{+}$, yield a constant current density with the same versus of $\mathbf{E}$ and a constant heat flux with the opposite versus of $\mathbf{g}$, expressed by

$$
\mathbf{J}(t)=\nu_{\infty}^{(\alpha)} \mathbf{E}, \quad \mathbf{q}(t)=-\nu_{\infty}^{(k)} \mathbf{g}
$$

due to (3.10).

Following [14, where only the kernel $\alpha$ has been considered, the inverse Fourier transform of $k_{c}(\omega)$ yields

$$
k(t)=\frac{2}{\pi} \int_{0}^{+\infty} k_{c}(\omega) \cos (\omega t) d \omega, \quad k(0)=\frac{2}{\pi} \int_{0}^{+\infty} k_{c}(\omega) d \omega>0
$$

by virtue of $(3.9)_{2}$. Hence we get

$$
k(0)-k(t)=\frac{2}{\pi} \int_{0}^{+\infty} k_{c}(\omega)[1-\cos (\omega t)] d \omega>0,
$$

from which it follows that $k^{\prime}(0) \leq 0$.

The hypotheses assumed for $k$ give the relation

$$
k_{s}^{\prime}(\omega)=-\omega k_{c}(\omega)
$$

hence, if $k^{\prime \prime} \in L^{2}\left(R^{+}\right)$and $\left|k^{\prime}(0)\right|<+\infty$, it follows that

$$
\lim _{\omega \rightarrow \infty} \omega k_{s}^{\prime}(\omega)=-\lim _{\omega \rightarrow \infty} \omega^{2} k_{c}(\omega)=k^{\prime}(0) \leq 0
$$

by virtue of $(3.9)_{2}$; the last inequality coincides with $(3.13)_{2}$.

The analogous results relative to the kernel $\alpha$ have been derived in [14] and coincide with all these; therefore, all the relations from (3.12) to (3.15) can be rewritten in terms of $\alpha, \alpha^{\prime}, \alpha^{\prime \prime}$ by simply changing $k$ with $\alpha$.

Taking into account $(3.13)_{2}$ and the analogous relation $\alpha^{\prime}(0) \leq 0$, we assume

$$
\alpha^{\prime}(0)<0, \quad k^{\prime}(0)<0 .
$$

4. Position of the problem: Continuation and equivalent histories. The constitutive equations (2.1)-(2.2), which we have assumed for $\mathcal{B}$, characterize a simple material [21, 22]; therefore the behaviour of our material can be described in terms of states and processes.

Thus, as we have already observed at the beginning of the previous section, the conductor $\mathcal{B}$ can be characterized by the state $\sigma(t)=\left(\mathbf{E}(t), \mathbf{H}(t), \vartheta(t), \mathbf{E}^{t}, \mathbf{g}^{t}\right) \in \Sigma$, the set of the admissible states, and by the process $P(\tau)=\left(\dot{\mathbf{E}}_{P}(\tau), \dot{\mathbf{H}}_{P}(\tau), \dot{\vartheta}_{P}(\tau), \mathbf{g}_{P}(\tau)\right)$, which is an integrable function defined on the time interval $[0, d)$ and expressed by the time derivatives of the electric and magnetic fields as well as of the temperature, together with the temperature gradient, each of which is assigned at any point of $[0, d)$. In particular, for example, $\dot{\mathbf{E}}_{P}(\tau)$ denotes the time derivative of $\mathbf{E}_{P}$ at $\tau \in[0, d)$. We have denoted by $d<+\infty$ the duration of the process $P \in \Pi$, the set of all admissible processes. We shall consider the restriction $P_{\left[t_{1}, t_{2}\right)}$ of the process $P$ of duration $d$, when we shall apply $P$ only in the interval $\left[t_{1}, t_{2}\right) \subset[0, d)$. We introduce the evolution function $\rho: \Sigma \times \Pi \rightarrow \Sigma$ as the function which maps an initial state $\sigma^{i} \in \Sigma$ and process $P \in \Pi$ into the final $\sigma^{f}=\rho\left(\sigma^{i}, P\right) \in \Sigma$. Moreover, when the restriction $P_{[0, t)}$ is considered for any $t \in[0, d]$, 
we have $\sigma(t)=\rho\left(\sigma(0), P_{[0, t)}\right)$, and, when $\sigma(d)=\rho(\sigma(0), P)=\sigma(0)$, the pair $(\sigma, P)$ is said to be a cycle.

Since in the sequel we shall be concerned with continuations of histories with processes, we must give the definition of such a continuation.

First, we consider the case when a process is applied at time $t=0$. In this case, denoting by $d$ the duration of the process, the generic instant in the time interval, where the process is defined, coincides with $t$ and we have $t \in[0, d)$. The process, expressed by $P(t)=\left(\dot{\mathbf{E}}_{P}(t), \dot{\mathbf{H}}_{P}(t), \dot{\vartheta}_{P}(t), \mathbf{g}_{P}(t)\right) \forall t \in[0, d)$, is applied at time $t=0$, when the initial state is $\sigma(0)=\left(\mathbf{E}_{*}(0), \mathbf{H}_{*}(0), \vartheta_{*}(0), \mathbf{E}_{*}^{0}, \mathbf{g}_{*}^{0}\right)$. It induces the set of the following states corresponding to every time $t \in(0, d]$, denoted by $\sigma(t)=\left(\mathbf{E}(t), \mathbf{H}(t), \vartheta(t), \mathbf{E}^{t}, \mathbf{g}^{t}\right)$ and defined by

$$
\begin{aligned}
\mathbf{E}(t) & =\mathbf{E}_{*}(0)+\int_{0}^{t} \dot{\mathbf{E}}_{P}(s) d s, \quad \mathbf{H}(t)=\mathbf{H}_{*}(0)+\int_{0}^{t} \dot{\mathbf{H}}_{P}(s) d s, \\
\vartheta(t) & =\vartheta_{*}(0)+\int_{0}^{t} \dot{\vartheta}_{P}(s) d s, \\
\mathbf{E}^{t}(\tau) & =\left\{\begin{array}{ll}
\mathbf{E}(t-\tau), & 0<\tau \leq t, \\
\mathbf{E}_{*}^{0}(\tau-t), & \tau>t,
\end{array} \mathbf{g}^{t}(\tau)= \begin{cases}\mathbf{g}_{P}(t-\tau), & 0<\tau \leq t, \\
\mathbf{g}_{*}^{0}(\tau-t), & \tau>t .\end{cases} \right.
\end{aligned}
$$

Here we have omitted the subscript $P$ in the functions present in $\sigma(t)$ and at the left-hand sides of (4.1)-(4.2).

Then, we consider the case when the process is applied at the generic time $t$. Now, it is given the initial state $\sigma(t)=\left(\mathbf{E}(t), \mathbf{H}(t), \vartheta(t), \mathbf{E}^{t}, \mathbf{g}^{t}\right)$, that is, the instantaneous values of the magnetic field $\mathbf{H}(t)$ and of the temperature $\vartheta(t)$ with the history $\mathbf{E}$ : $(-\infty, t] \rightarrow \mathbf{R}^{3}$ and the past history $\mathbf{g}^{t}(s)=\mathbf{g}(t-s) \forall s \in \mathbf{R}^{++}$. We relate a process $P(\tau)=\left(\dot{\mathbf{E}}_{P}(\tau), \dot{\mathbf{H}}_{P}(\tau), \dot{\vartheta}_{P}(\tau), \mathbf{g}_{P}(\tau)\right) \forall \tau \in[0, d), d$ being the duration of the process applied at time $t$, to

$$
\begin{array}{ll}
\mathbf{E}_{P}:(0, d] \rightarrow \mathbf{R}^{3}, & \mathbf{E}_{P}(\tau)=\mathbf{E}(t)+\int_{0}^{\tau} \dot{\mathbf{E}}_{P}(\xi) d \xi, \\
\mathbf{H}_{P}:(0, d] \rightarrow \mathbf{R}^{3}, & \mathbf{H}_{P}(\tau)=\mathbf{H}(t)+\int_{0}^{\tau} \dot{\mathbf{H}}_{P}(\xi) d \xi, \\
\vartheta_{P}:(0, d] \rightarrow \mathbf{R}, & \vartheta_{P}(\tau)=\vartheta(t)+\int_{0}^{\tau} \dot{\vartheta}_{P}(\xi) d \xi,
\end{array}
$$

defined for any $\tau \in(0, d]$, where $\tau \neq 0$ to distinguish the values assumed by the fields during the application of the process from their instantaneous values at time $t$. Moreover, in particular,

$$
\mathbf{g}_{P}:[0, d) \rightarrow \mathbf{R}^{3}, \quad \mathbf{g}_{P}(\tau)=\mathbf{g}(t+\tau)
$$

is assigned by $P$.

Therefore, the final value of the electric field at each instant $\tau^{\prime} \equiv t+\tau \leq t+d$ is denoted by $\mathbf{E}_{f}\left(\tau^{\prime}\right)=\left(\mathbf{E}_{P} * \mathbf{E}\right)\left(\tau^{\prime}\right)$, it depends upon $\mathbf{E}^{t}, \dot{\mathbf{E}}_{P}$ and, moreover, it is related to $\mathbf{E}_{P}(d),\left(\mathbf{E}_{P} * \mathbf{E}\right)^{t+d}$. Its expression is the following:

$$
\mathbf{E}_{f}(t+d-s)=\left(\mathbf{E}_{P} * \mathbf{E}\right)(t+d-s)= \begin{cases}\mathbf{E}_{P}(d-s), & 0 \leq s<d \\ \mathbf{E}(t+d-s), & s \geq d\end{cases}
$$


where $*$ denotes the continuation of histories with some process. Analogously, the continuation of the past history of the temperature gradient is defined at each $\tau^{\prime} \equiv t+\tau<t+d$ and denoted by $\mathbf{g}_{f}\left(\tau^{\prime}\right)=\left(\mathbf{g}_{P} * \mathbf{g}\right)\left(\tau^{\prime}\right)$, which depends upon $\mathbf{g}^{t}, \mathbf{g}_{P}$ and is given by

$$
\mathbf{g}_{f}(t+d-s)=\left(\mathbf{g}_{P} * \mathbf{g}\right)(t+d-s)= \begin{cases}\mathbf{g}_{P}(d-s), & 0<s \leq d, \\ \mathbf{g}(t+d-s), & s>d,\end{cases}
$$

due to (4.7), too.

The constitutive equations (2.2) allow us to consider two linear functionals $\tilde{\mathbf{J}}: \Gamma_{\alpha} \rightarrow$ $\mathbf{R}^{3}$ and $\tilde{\mathbf{q}}: \Gamma_{k} \rightarrow \mathbf{R}^{3}$, which give the set of the possible current densities and one of the possible heat fluxes in correspondence to different histories of the electric field and of the temperature gradient, respectively. They are defined by

$$
\tilde{\mathbf{J}}\left(\mathbf{E}^{t}\right)=\int_{0}^{+\infty} \alpha(s) \mathbf{E}^{t}(s) d s, \quad \tilde{\mathbf{q}}\left(\mathbf{g}^{t}\right)=-\int_{0}^{+\infty} k(s) \mathbf{g}^{t}(s) d s,
$$

where $\mathbf{E}^{t} \in \Gamma_{\alpha}$ and $\mathbf{g}^{t} \in \Gamma_{k}$.

The introduction of these two functionals allows us to give the following definition.

Definition 4.1. Let $\left(\mathbf{E}_{j}(t), \mathbf{E}_{j}^{t}\right), j=1,2$, be two couples of histories of the electric field and $\mathbf{g}_{j}^{t}, j=1,2$, two past histories of the temperature gradient, corresponding to the same instantaneous values $\mathbf{H}(t)$ of the magnetic field and $\vartheta(t)$ of the temperature. They are called equivalent if for every $\tau>0$ we have

$$
\mathbf{E}_{1}(t)=\mathbf{E}_{2}(t), \quad \tilde{\mathbf{J}}\left(\left(\mathbf{E}_{P} * \mathbf{E}_{1}\right)^{t+\tau}\right)=\tilde{\mathbf{J}}\left(\left(\mathbf{E}_{P} * \mathbf{E}_{2}\right)^{t+\tau}\right)
$$

for every $\mathbf{E}_{P}:(0, \tau] \rightarrow \mathbf{R}^{3}$ and

$$
\tilde{\mathbf{q}}\left(\left(\mathbf{g}_{P} * \mathbf{g}_{1}\right)^{t+\tau}\right)=\tilde{\mathbf{q}}\left(\left(\mathbf{g}_{P} * \mathbf{g}_{2}\right)^{t+\tau}\right)
$$

for every $\mathbf{g}_{P}:[0, \tau) \rightarrow \mathbf{R}^{3}$, whatever may be $\mathbf{H}_{P}:(0, \tau] \rightarrow \mathbf{R}^{3}$ and $\vartheta_{P}:(0, \tau] \rightarrow \mathbf{R}$.

We note that for any process the values $\mathbf{H}_{P}(\tau)$ and $\vartheta_{P}(\tau)$ are independent both of the two couples of histories $\left(\mathbf{E}_{1}(t), \mathbf{E}_{1}^{t}\right)$ and $\left(\mathbf{E}_{2}(t), \mathbf{E}_{2}^{t}\right)$ and of the two past histories $\mathbf{g}_{1}^{t}$ and $\mathbf{g}_{2}^{t}$. Moreover, since we have the same $\mathbf{g}_{P}$ defined on $[0, \tau)$ for both the past histories $\mathbf{g}_{1}^{t}$ and $\mathbf{g}_{2}^{t}$, in particular, we have in both of these cases the same initial value $\mathbf{g}_{P}(0)=\mathbf{g}(t)$ due to (4.7). Finally, for the equivalence of two couples of histories both the same electric current density and the same heat flux, i.e., the same response of the material, are required.

In particular, if we consider the history $\left(\mathbf{0}, \mathbf{E}^{t}\right)$, that is, the history characterized by a zero instantaneous value for the electric current density and the past history $\mathbf{g}^{t}$, they are equivalent to the zero history $\left(\mathbf{0}, \mathbf{0}^{\dagger}\right)$ of $\mathbf{E}$ and the zero past history $\mathbf{0}^{\dagger}$ of $\mathbf{g}$ if

$$
\int_{0}^{+\infty} \alpha(\tau+\xi) \mathbf{E}^{t}(\xi) d \xi=\int_{\tau}^{+\infty} \alpha(s) \mathbf{E}^{t+\tau}(s) d s=\mathbf{0}
$$

and

$$
\int_{0}^{+\infty} k(\tau+\xi) \mathbf{g}^{t}(\xi) d \xi=\int_{\tau}^{+\infty} k(s) \mathbf{g}^{t+\tau}(s) d s=\mathbf{0} .
$$

We observe that both the conditions (4.11)-(4.12) and the relations (4.13)-(4.14) give an equivalence relation, which allows us to state that two past histories $\mathbf{E}_{1}^{t}, \mathbf{g}_{1}^{t}$ and $\mathbf{E}_{2}^{t}, \mathbf{g}_{2}^{t}$ are equivalent in the sense of Definition 4.1 if their differences $\mathbf{E}^{t}=\mathbf{E}_{1}^{t}-\mathbf{E}_{2}^{t}, \mathbf{g}^{t}=\mathbf{g}_{1}^{t}-\mathbf{g}_{2}^{t}$ satisfy (4.13)-(4.14), respectively. 
Furthermore, taking into account Noll's definition of state 22, we can state that two couples of histories $\left(\mathbf{E}_{j}(t), \mathbf{E}_{j}^{t}\right), j=1,2$, with $\mathbf{E}_{1}(t)=\mathbf{E}_{2}(t)$, and two past histories $\mathbf{g}_{j}^{t}, j=1,2$, such that the differences $\mathbf{E}_{1}^{t}-\mathbf{E}_{2}^{t}, \mathbf{g}_{1}^{t}-\mathbf{g}_{2}^{t}$ satisfy (4.13)-(4.14), give the same state $\sigma(t)$, with the fixed values of $\mathbf{H}(t)$ and $\vartheta(t)$. Thus, the state $\sigma(t)$ can be seen as the "minimum" set of variables which yield a univocal relation between $\left(\dot{\mathbf{E}}_{P}(\cdot), \dot{\mathbf{H}}_{P}(\cdot), \dot{\vartheta}_{P}(\cdot), \mathbf{g}_{P}(\cdot)\right)$, defined in $[0, \tau)$, and $(\mathbf{D}(t+\tau), \mathbf{B}(t+\tau), \mathbf{J}(t+\tau), \mathbf{q}(t+\tau))$, where, due to (2.1), $\mathbf{D}(t+\tau)$ is expressed by (4.4) and (4.6), $\mathbf{B}(t+\tau)$ by (4.5) and $\mathbf{J}(t+\tau)=\tilde{\mathbf{J}}\left(\left(\mathbf{E}_{P} * \mathbf{E}\right)^{t+\tau}\right), \mathbf{q}(t+\tau)=\tilde{\mathbf{q}}\left(\left(\mathbf{g}_{P} * \mathbf{g}\right)^{t+\tau}\right) \forall \tau>0$. Hence, it follows that the state $\sigma$ becomes an element of $\Sigma=\mathbf{R}^{3} \times \mathbf{R}^{3} \times \mathbf{R} \times\left(\Gamma_{\alpha} / \Gamma_{\alpha_{0}}\right) \times\left(\Gamma_{k} / \Gamma_{k_{0}}\right)$, where $\Gamma_{\alpha} / \Gamma_{\alpha_{0}}$ and $\Gamma_{k} / \Gamma_{k_{0}}$ are the usual quotient spaces, $\Gamma_{\alpha_{0}} \subset \Gamma_{\alpha}$ and $\Gamma_{k_{0}} \subset \Gamma_{k}$ being the subsets of the past histories which satisfy (4.13) and (4.14), respectively.

Therefore, it follows that the boundednesses of $\mathbf{J}$ and $\mathbf{q}$ are related to this definition of the state. We shall consider the boundedness of the work, too.

5. Thermoelectromagnetic work. As we have already stated in Sec. 2, the inequality (2.11) expresses the local form of the second law of thermodynamics for the thermoelectromagnetic conductor $\mathcal{B}$. It must hold for any process $P$, which, starting from the initial state $\sigma$, yields the final state $\sigma^{f} \equiv \sigma$, i.e. for any cycle $(\sigma, P)$; moreover, it gives the thermoelectromagnetic work done on the process $P$ [9, 11].

Denoting by $\sigma^{i}(t)=\left(\mathbf{E}_{i}(t), \mathbf{H}_{i}(t), \vartheta_{i}(t), \mathbf{E}_{i}^{t}, \mathbf{g}_{i}^{t}\right)$ the initial state of $\mathcal{B}$, we consider the process $P(\tau)=\left(\dot{\mathbf{E}}_{P}(\tau), \dot{\mathbf{H}}_{P}(\tau), \dot{\vartheta}_{P}(\tau), \mathbf{g}_{P}(\tau)\right), \forall \tau \in[0, d)$, that is, with duration $d$ and related to the time interval $[t, t+d)$. The work done on $P$ is a function of the state and of the process and is expressed by

$$
\begin{aligned}
W\left(\sigma^{i}(t), P\right)=\tilde{W}\left(\mathbf{E}_{i}(t), \mathbf{H}_{i}(t), \vartheta_{i}(t), \mathbf{E}_{i}^{t}, \mathbf{g}_{i}^{t} ; \dot{\mathbf{E}}_{P}, \dot{\mathbf{H}}_{P}, \dot{\vartheta}_{P}, \mathbf{g}_{P}\right) \\
\quad=\int_{0}^{d}\left[\frac{c}{\Theta_{0}} \dot{\vartheta}_{P}(\tau) \vartheta_{P}(\tau)+\frac{1}{\varepsilon} \dot{\mathbf{D}}\left(\mathbf{E}_{P}(\tau), \vartheta_{P}(\tau)\right) \cdot \mathbf{D}\left(\mathbf{E}_{P}(\tau), \vartheta_{P}(\tau)\right)+\frac{1}{\mu} \dot{\mathbf{B}}\left(\mathbf{H}_{P}(\tau)\right)\right. \\
\left.\quad \cdot \mathbf{B}\left(\mathbf{H}_{P}(\tau)\right)+\tilde{\mathbf{J}}\left(\left(\mathbf{E}_{P} * \mathbf{E}_{i}\right)^{t+\tau}\right) \cdot \mathbf{E}_{P}(\tau)-\frac{1}{\Theta_{0}} \tilde{\mathbf{q}}\left(\left(\mathbf{g}_{P} * \mathbf{g}_{i}\right)^{t+\tau}\right) \cdot \mathbf{g}_{P}(\tau)\right] d \tau,
\end{aligned}
$$

where the integral is expressed as a function of $\tau \in[0, d)$ and consequently $\mathbf{E}_{P}(\tau), \mathbf{H}_{P}(\tau)$, $\vartheta_{P}(\tau)$ are given by (4.4)-(4.6), $\mathbf{g}_{P}(\tau)$ is assigned by $P$ with (4.7) and the continuations $\mathbf{E}_{P} * \mathbf{E}_{i}, \mathbf{g}_{P} * \mathbf{g}_{i}$ are defined by (4.8)-(4.9).

Another equivalent form of this work is the following:

$$
\begin{aligned}
& W\left(\sigma^{i}(t), P\right)=\int_{t}^{t+d}\left[\frac{c}{\Theta_{0}} \dot{\vartheta}(\xi) \vartheta(\xi)+\frac{1}{\varepsilon} \dot{\mathbf{D}}(\mathbf{E}(\xi), \vartheta(\xi)) \cdot \mathbf{D}(\mathbf{E}(\xi), \vartheta(\xi))\right. \\
& \left.+\frac{1}{\mu} \dot{\mathbf{B}}(\mathbf{H}(\xi)) \cdot \mathbf{B}(\mathbf{H}(\xi))+\tilde{\mathbf{J}}\left(\left(\mathbf{E}_{P} * \mathbf{E}_{i}\right)^{\xi}\right) \cdot \mathbf{E}(\xi)-\frac{1}{\Theta_{0}} \tilde{\mathbf{q}}\left(\left(\mathbf{g}_{P} * \mathbf{g}_{i}\right)^{\xi}\right) \cdot \mathbf{g}(\xi)\right] d \xi
\end{aligned}
$$

where the integral is written in terms of $\xi \in[t, t+d)$.

This work done on the process $P$, of duration $d$, which yields the continuation from the initial state, denoted simply by $\sigma(t)=\left(\mathbf{E}(t), \mathbf{H}(t), \vartheta(t), \mathbf{E}^{t}, \mathbf{g}^{t}\right)$, is given by (5.1) or 
(5.2). Taking into account (2.1) and (4.10), from (5.2) we obtain

$$
\begin{aligned}
W(\sigma(t), P)=\frac{1}{2}[ & \left.\frac{c}{\Theta_{0}} \vartheta^{2}(t+d)+\frac{1}{\varepsilon} \mathbf{D}^{2}(t+d)+\frac{1}{\mu} \mathbf{B}^{2}(t+d)\right] \\
& -\frac{1}{2}\left[\frac{c}{\Theta_{0}} \vartheta^{2}(t)+\frac{1}{\varepsilon} \mathbf{D}^{2}(t)+\frac{1}{\mu} \mathbf{B}^{2}(t)\right] \\
& +\int_{t}^{t+d}\left[\int_{0}^{+\infty} \alpha(s) \mathbf{E}^{\xi}(s) d s \cdot \mathbf{E}(\xi)+\frac{1}{\Theta_{0}} \int_{0}^{+\infty} k(s) \mathbf{g}^{\xi}(s) d s \cdot \mathbf{g}(\xi)\right] d \xi,
\end{aligned}
$$

where we have put $\mathbf{E}^{\xi}=\left(\mathbf{E}_{P} * \mathbf{E}\right)^{\xi}$ and $\mathbf{g}^{\xi}=\left(\mathbf{g}_{P} * \mathbf{g}\right)^{\xi}$, which are given by (4.8) and (4.9), respectively.

It is interesting to examine the case when the initial state is characterized by null histories for the electric field and the temperature gradient. Thus, let us consider the null history $\left(\mathbf{0}, \mathbf{0}^{\dagger}\right)$ of the electric field and the null past history $\mathbf{0}^{\dagger}$ of the temperature gradient, where $\mathbf{0}^{\dagger}(s)=\mathbf{0} \forall s>0$; we now take the initial instant as $t=0$ for simplicity. Then, assuming $\mathbf{H}(0)=\mathbf{0}$ and $\vartheta(0)=0$, we apply a process $P$, which gives $\mathbf{g}_{P}:[0, d) \rightarrow \mathbf{R}^{3}$ and is related to the functions $\mathbf{E}_{P}:(0, d] \rightarrow \mathbf{R}^{3}, \mathbf{H}_{P}:(0, d] \rightarrow \mathbf{R}^{3}, \vartheta_{P}:(0, d] \rightarrow \mathbf{R}$ and the continuations of the two histories of $\mathbf{E}$ and $\mathbf{g}$. Under these hypotheses, the ensuing fields, denoted by $\left(\mathbf{E}_{0}(t), \mathbf{H}_{0}(t), \vartheta_{0}(t), \mathbf{E}_{0}^{t}, \mathbf{g}_{0}^{t}\right)$ with $t \in(0, d]$, by virtue of (4.4)-(4.9), assume the form

$$
\begin{aligned}
\mathbf{E}_{0}(t) & =\int_{0}^{t} \dot{\mathbf{E}}_{P}(\tau) d \tau, \quad \mathbf{H}_{0}(t)=\int_{0}^{t} \dot{\mathbf{H}}_{P}(\tau) d \tau, \\
\vartheta_{0}(t) & =\int_{0}^{t} \dot{\vartheta}_{P}(\tau) d \tau
\end{aligned}
$$

and

$$
\mathbf{E}_{0}^{t}(s)=\left\{\begin{array}{ll}
\int_{0}^{t-s} \dot{\mathbf{E}}_{P}(\tau) d \tau, & 0<s \leq t, \\
\mathbf{0}, & s>t,
\end{array} \mathbf{g}_{0}^{t}(s)= \begin{cases}\mathbf{g}_{P}(t-s), & 0<s \leq t, \\
\mathbf{0}, & s>t .\end{cases}\right.
$$

Denoting by

$$
\sigma_{0}(0)=\left(\mathbf{0}, \mathbf{0}, 0, \mathbf{0}^{\dagger}, \mathbf{0}^{\dagger}\right)
$$

the initial state which we have introduced (that is, the one corresponding to null values of the interested functions at $t=0$ and to null past histories for $\mathbf{E}$ and $\mathbf{g}$ ), work done on a process $P=\left(\dot{\mathbf{E}}_{P}, \dot{\mathbf{H}}_{P}, \dot{\vartheta}_{P}, \mathbf{g}_{P}\right)$, applied at $t=0$, is given by (5.2), which now reduces to

$$
\begin{array}{r}
W\left(\sigma_{0}(0), P\right)=\int_{0}^{d}\left[\frac{c}{\Theta_{0}} \dot{\vartheta}_{0}(t) \vartheta_{0}(t)+\frac{1}{\varepsilon} \dot{\mathbf{D}}\left(\mathbf{E}_{0}(t), \vartheta_{0}(t)\right) \cdot \mathbf{D}\left(\mathbf{E}_{0}(t), \vartheta_{0}(t)\right)\right. \\
\left.+\frac{1}{\mu} \dot{\mathbf{B}}\left(\mathbf{H}_{0}(t)\right) \cdot \mathbf{B}\left(\mathbf{H}_{0}(t)\right)+\tilde{\mathbf{J}}\left(\mathbf{E}_{0}^{t}\right) \cdot \mathbf{E}_{0}(t)-\frac{1}{\Theta_{0}} \tilde{\mathbf{q}}\left(\mathbf{g}_{0}^{t}\right) \cdot \mathbf{g}_{0}(t)\right] d t,
\end{array}
$$

where, in particular, $\mathbf{g}_{0}(t) \equiv \mathbf{g}_{P}(t)$ is the assigned temperature gradient in $P$.

This relation allows us to introduce the following definition. 
Definition 5.1. A process $P(t)=\left(\dot{\mathbf{E}}_{P}(t), \dot{\mathbf{H}}_{P}(t), \dot{\vartheta}_{P}(t), \mathbf{g}_{P}(t)\right)$, defined for any $t \in$ $[0, d)$, starting from the state $\sigma_{0}(0)$ given by $(5.7)$, is said to be a finite work process if the work done on $P$ and expressed by (5.8) with (5.4)-(5.6) satisfies

$$
W\left(\sigma_{0}(0), P\right)<+\infty \text {. }
$$

We have the following result.

LEMmA 5.1. The work done on any process, which is a finite work process in the sense of Definition 5.1, is positive.

Proof. The work done on a process $P$, of duration $d$, starting from the state $\sigma_{0}(0)$ defined by (5.7), is expressed by (5.8). It is finite if $P$ is a finite work process in the sense of Definition 5.1; therefore, (5.9) is satisfied. Let us assume that the ensuing fields, present in (5.8) and given by (5.4)-(5.6), vanish for any $t>d$, the duration of $P$. Thus, taking into account the constitutive equations (2.1) and (4.10) with (5.6), (5.8) assumes the form

$$
\begin{aligned}
W\left(\sigma_{0}(0), P\right)= & \frac{1}{2}\left[\frac{c}{\Theta_{0}} \vartheta_{0}^{2}(d)+\frac{1}{\varepsilon} \mathbf{D}_{0}^{2}(d)+\frac{1}{\mu} \mathbf{B}_{0}^{2}(d)\right] \\
& +\int_{0}^{+\infty}\left[\int_{0}^{+\infty} \alpha(s) \mathbf{E}_{0}^{t}(s) d s \cdot \mathbf{E}_{0}(t)+\frac{1}{\Theta_{0}} \int_{0}^{+\infty} k(s) \mathbf{g}_{0}^{t}(s) d s \cdot \mathbf{g}_{0}(t)\right] d t,
\end{aligned}
$$

which, by virtue of Plancherel's theorem, becomes

$$
\begin{aligned}
W\left(\sigma_{0}(0), P\right)= & \frac{1}{2}\left[\frac{c}{\Theta_{0}} \vartheta_{0}^{2}(d)+\frac{1}{\varepsilon} \mathbf{D}_{0}^{2}(d)+\frac{1}{\mu} \mathbf{B}_{0}^{2}(d)\right] \\
& +\frac{1}{2 \pi} \int_{-\infty}^{+\infty}\left[\alpha_{F}(\omega) \mathbf{E}_{0_{F}}(\omega) \cdot \mathbf{E}_{0_{F}}^{*}(\omega)+\frac{1}{\Theta_{0}} k_{F}(\omega) \mathbf{g}_{0_{F}}(\omega) \cdot \mathbf{g}_{0_{F}}^{*}(\omega)\right] d \omega
\end{aligned}
$$

where ${ }^{*}$ denotes the complex conjugate.

To prove the positiveness of this expression, it is enough to consider the sign of the integral present in it.

To this end, we observe that (on the ground of the properties of the Fourier transforms, recalled in Sec. 3) in (5.11) the functions, whose Fourier transforms must be considered, are defined on $\mathbf{R}^{+}$and considered equal to zero on $\mathbf{R}^{--}$. Therefore, their cosine and sine transforms, in terms of which the Fourier transforms can be expressed on the ground of (3.7), are even and odd functions. 
Thus, the integral at the right-hand side of (5.11) can be changed as follows:

$$
\begin{aligned}
\frac{1}{2 \pi} \int_{-\infty}^{+\infty} & {\left[\alpha_{F}(\omega) \mathbf{E}_{0_{F}}(\omega) \cdot \mathbf{E}_{0_{F}}^{*}(\omega)+\frac{1}{\Theta_{0}} k_{F}(\omega) \mathbf{g}_{0_{F}}(\omega) \cdot \mathbf{g}_{0_{F}}^{*}(\omega)\right] d \omega } \\
= & \frac{1}{2 \pi} \int_{-\infty}^{+\infty}\left[\left(\alpha_{c}-i \alpha_{s}\right)\left(\mathbf{E}_{0_{c}}-i \mathbf{E}_{0_{s}}\right) \cdot\left(\mathbf{E}_{0_{c}}+i \mathbf{E}_{0_{s}}\right)\right. \\
& \left.\quad+\frac{1}{\Theta_{0}}\left(k_{c}-i k_{s}\right)\left(\mathbf{g}_{0_{c}}-i \mathbf{g}_{0_{s}}\right) \cdot\left(\mathbf{g}_{0_{c}}+i \mathbf{g}_{0_{s}}\right)\right] d \omega \\
= & \frac{1}{2 \pi} \int_{-\infty}^{+\infty}\left\{\alpha_{c}(\omega)\left[\mathbf{E}_{0_{c}}^{2}(\omega)+\mathbf{E}_{0_{s}}^{2}(\omega)\right]+\frac{1}{\Theta_{0}} k_{c}(\omega)\left[\mathbf{g}_{0_{c}}^{2}(\omega)+\mathbf{g}_{0_{s}}^{2}(\omega)\right]\right\} d \omega>0,
\end{aligned}
$$

where the inequality holds due to (3.9).

Generally, a process $P$ has a finite duration, $d<+\infty$. However, we can consider $P$ defined on $\mathbf{R}^{+}$, if we suppose that $P(\tau)=\left(\dot{\mathbf{E}}_{P}(\tau), \dot{\mathbf{H}}_{P}(\tau), \dot{\vartheta}_{P}(\tau), \mathbf{g}_{P}(\tau)\right)=(\mathbf{0}, \mathbf{0}, 0, \mathbf{0})$ $\forall \tau \geq d$. If we also assume that $\mathbf{E}_{P}(\tau)=\mathbf{0}, \mathbf{H}_{P}(\tau)=\mathbf{0}, \vartheta_{P}(\tau)=0 \forall \tau>d$, (5.8), again using the subscript $P$ in the integrals with the histories, becomes

$$
\begin{aligned}
W\left(\sigma_{0}(0), P\right)=\int_{0}^{d}\left[\frac{c}{\Theta_{0}} \dot{\vartheta}_{0}(\xi) \vartheta_{0}(\xi)+\frac{1}{\varepsilon} \dot{\mathbf{D}}_{0}(\xi) \cdot \mathbf{D}_{0}(\xi)+\frac{1}{\mu} \dot{\mathbf{B}}_{0}(\xi) \cdot \mathbf{B}_{0}(\xi)\right] d \xi \\
\quad+\int_{0}^{+\infty}\left[\int_{0}^{+\infty} \alpha(s) \mathbf{E}_{P}(\eta-s) d s \cdot \mathbf{E}_{P}(\eta)+\frac{1}{\Theta_{0}} \int_{0}^{+\infty} k(s) \mathbf{g}_{P}(\eta-s) d s \cdot \mathbf{g}_{P}(\eta)\right] d \eta \\
=\frac{1}{2}\left[\frac{c}{\Theta_{0}} \vartheta_{0}^{2}(d)+\frac{1}{\varepsilon} \mathbf{D}_{0}^{2}(d)+\frac{1}{\mu} \mathbf{B}_{0}^{2}(d)\right]+\int_{0}^{+\infty}\left[\int_{0}^{\eta} \alpha(s) \mathbf{E}_{P}(\eta-s) d s \cdot \mathbf{E}_{P}(\eta)\right. \\
\left.\quad+\frac{1}{\Theta_{0}} \int_{0}^{\eta} k(s) \mathbf{g}_{P}(\eta-s) d s \cdot \mathbf{g}_{P}(\eta)\right] d \eta
\end{aligned}
$$

since for any $s>\eta$ we have the null histories. The last two integrals in (5.13) can be changed as follows:

$$
\begin{aligned}
\int_{0}^{+\infty} & {\left[\int_{0}^{\eta} \alpha(s) \mathbf{E}_{P}(\eta-s) d s \cdot \mathbf{E}_{P}(\eta)+\frac{1}{\Theta_{0}} \int_{0}^{\eta} k(s) \mathbf{g}_{P}(\eta-s) d s \cdot \mathbf{g}_{P}(\eta)\right] d \eta } \\
= & \int_{0}^{+\infty}\left[\int_{0}^{\eta} \alpha(\eta-\rho) \mathbf{E}_{P}(\rho) d \rho \cdot \mathbf{E}_{P}(\eta)+\frac{1}{\Theta_{0}} \int_{0}^{\eta} k(\eta-\rho) \mathbf{g}_{P}(\rho) d \rho \cdot \mathbf{g}_{P}(\eta)\right] d \eta \\
= & \frac{1}{2}\left[\int_{0}^{+\infty} \int_{0}^{+\infty} \alpha(|\eta-\rho|) \mathbf{E}_{P}(\rho) \cdot \mathbf{E}_{P}(\eta) d \rho d \eta\right. \\
& \left.+\frac{1}{\Theta_{0}} \int_{0}^{+\infty} \int_{0}^{+\infty} k(|\eta-\rho|) \mathbf{g}_{P}(\rho) \cdot \mathbf{g}_{P}(\eta) d \rho d \eta\right]
\end{aligned}
$$


Substituting the last expression after applying Plancherel's theorem and taking into account that both $\alpha(|\eta-\rho|)$ and $k(|\eta-\rho|)$ are even functions whose Fourier transforms can be expressed in terms of their Fourier cosine transforms, (5.13) assumes the following form:

$$
\begin{aligned}
W\left(\sigma_{0}(0), P\right)= & \frac{1}{2}\left[\frac{c}{\Theta_{0}} \vartheta_{0}^{2}(d)+\frac{1}{\varepsilon} \mathbf{D}_{0}^{2}(d)+\frac{1}{\mu} \mathbf{B}_{0}^{2}(d)\right] \\
+\frac{1}{2 \pi} \int_{-\infty}^{+\infty}\left[\alpha_{c}(\omega) \mathbf{E}_{P+}(\omega)\right. & \left.\cdot \mathbf{E}_{P+}^{*}(\omega)+\frac{1}{\Theta_{0}} k_{c}(\omega) \mathbf{g}_{P+}(\omega) \cdot \mathbf{g}_{P+}^{*}(\omega)\right] d \omega .
\end{aligned}
$$

Let

$$
\tilde{H}_{\alpha}\left(\mathbf{R}^{+}, \mathbf{R}^{3}\right)=\left\{\mathbf{E}: \mathbf{R}^{+} \rightarrow \mathbf{R}^{3} ; \quad \int_{-\infty}^{+\infty} \alpha_{c}(\omega) \mathbf{E}_{+}(\omega) \cdot \mathbf{E}_{+}^{*}(\omega) d \omega<+\infty\right\}
$$

and

$$
\tilde{H}_{k}\left(\mathbf{R}^{+}, \mathbf{R}^{3}\right)=\left\{\mathbf{g}: \mathbf{R}^{+} \rightarrow \mathbf{R}^{3} ; \quad \int_{-\infty}^{+\infty} k_{c}(\omega) \mathbf{g}_{+}(\omega) \cdot \mathbf{g}_{+}^{*}(\omega) d \omega<+\infty\right\}
$$

denote the sets of the functions related to finite work processes. When in these spaces the following inner products $\left(\mathbf{E}_{1}, \mathbf{E}_{2}\right)_{\alpha}=\int_{-\infty}^{+\infty} \alpha_{c}(\omega) \mathbf{E}_{1+}(\omega) \cdot \mathbf{E}_{2+}^{*}(\omega) d \omega$ and $\left(\mathbf{g}_{1}, \mathbf{g}_{2}\right)_{k}=$ $\int_{-\infty}^{+\infty} k_{c}(\omega) \mathbf{g}_{1+}(\omega) \cdot \mathbf{g}_{2+}^{*}(\omega) d \omega$ are introduced, we have in them the norms $\|\mathbf{E}\|_{\alpha}=(\mathbf{E}, \mathbf{E})_{\alpha}$ and $\|\mathbf{g}\|_{k}=(\mathbf{g}, \mathbf{g})_{k}$, respectively. The completion of these spaces with respect to these corresponding norms allows us to obtain the spaces of the processes, which, in particular, give $\mathbf{g}_{P}$ and are related to $\mathbf{E}_{P}$, that is, the Hilbert spaces which we denote by $H_{\alpha}\left(\mathbf{R}^{+}, \mathbf{R}^{3}\right)$ and $H_{k}\left(\mathbf{R}^{+}, \mathbf{R}^{3}\right)$.

Furthermore, the set of all admissible histories can be considered as the set of all the histories $\left(\mathbf{E}(t), \mathbf{E}^{t}\right)$ of the electric field and of the past histories $\mathbf{g}^{t}$ of the temperature gradient, such that the work done on any process, characterized by $\mathbf{g}_{P} \in H_{k}\left(\mathbf{R}^{+}, \mathbf{R}^{3}\right)$ and related to $\mathbf{E}_{P} \in H_{\alpha}\left(\mathbf{R}^{+}, \mathbf{R}^{3}\right)$, starting from the state corresponding to them, is finite. Thus, supposing again that for any $\tau \geq d(d<+\infty)$ the process is zero and corresponding to the values $\mathbf{E}_{P}(\tau)=\mathbf{0}, \mathbf{H}_{P}(\tau)=\mathbf{0}, \vartheta_{P}(\tau)=0 \forall \tau>d$, the work (5.1) is 
expressed, due to (4.8) and (4.9), by

$$
\begin{aligned}
W(\sigma(t), P)= & \frac{1}{2}\left[\frac{c}{\Theta_{0}} \vartheta_{P}^{2}(d)+\frac{1}{\varepsilon} \mathbf{D}^{2}\left(\mathbf{E}_{P}(d), \vartheta_{P}(d)\right)+\frac{1}{\mu} \mathbf{B}^{2}\left(\mathbf{H}_{P}(d)\right)\right] \\
& -\frac{1}{2}\left[\frac{c}{\Theta_{0}} \vartheta_{P}^{2}(0)+\frac{1}{\varepsilon} \mathbf{D}^{2}\left(\mathbf{E}_{P}(0), \vartheta_{P}(0)\right)+\frac{1}{\mu} \mathbf{B}^{2}\left(\mathbf{H}_{P}(0)\right)\right] \\
& +\int_{0}^{+\infty}\left[\int_{0}^{\tau} \alpha(s) \mathbf{E}_{P}(\tau-s) d s+\int_{\tau}^{+\infty} \alpha(s) \mathbf{E}(t+\tau-s) d s\right] \cdot \mathbf{E}_{P}(\tau) d \tau \\
& +\frac{1}{\Theta_{0}} \int_{0}^{+\infty}\left[\int_{0}^{\tau} k(s) \mathbf{g}_{P}(\tau-s) d s+\int_{\tau}^{+\infty} k(s) \mathbf{g}(t+\tau-s) d s\right] \cdot \mathbf{g}_{P}(\tau) d \tau \\
= & \frac{1}{2}\left[\frac{c}{\Theta_{0}} \vartheta_{P}^{2}(d)+\frac{1}{\varepsilon} \mathbf{D}^{2}\left(\mathbf{E}_{P}(d), \vartheta_{P}(d)\right)+\frac{1}{\mu} \mathbf{B}^{2}\left(\mathbf{H}_{P}(d)\right)\right] \\
& -\frac{1}{2}\left[\frac{c}{\Theta_{0}} \vartheta_{P}^{2}(0)+\frac{1}{\varepsilon} \mathbf{D}^{2}\left(\mathbf{E}_{P}(0), \vartheta_{P}(0)\right)+\frac{1}{\mu} \mathbf{B}^{2}\left(\mathbf{H}_{P}(0)\right)\right] \\
& +\int_{0}^{+\infty}\left[\int_{0}^{\tau} \alpha(s) \mathbf{E}_{P}(\tau-s) d s \cdot \mathbf{E}_{P}(\tau)\right. \\
& \left.\quad+\frac{1}{\Theta_{0}} \int_{0}^{\tau} k(s) \mathbf{g}_{P}(\tau-s) d s \cdot \mathbf{g}_{P}(\tau)\right] d \tau \\
& +\int_{0}^{+\infty}\left[\int_{0}^{+\infty} \alpha(\tau+\eta) \mathbf{E}^{t}(\eta) d \eta \cdot \mathbf{E}_{P}(\tau)\right. \\
& \left.\quad+\frac{1}{\Theta_{0}} \int_{0}^{+\infty} k(\tau+\eta) \mathbf{g}^{t}(\eta) d \eta \cdot \mathbf{g}_{P}(\tau)\right] d \tau .
\end{aligned}
$$

Putting

$$
\mathbf{I}_{(\alpha)}\left(\tau, \mathbf{E}^{t}\right)=-\int_{0}^{+\infty} \alpha(\tau+\eta) \mathbf{E}^{t}(\eta) d \eta, \mathbf{I}_{(k)}\left(\tau, \mathbf{g}^{t}\right)=-\int_{0}^{+\infty} k(\tau+\eta) \mathbf{g}^{t}(\eta) d \eta, \tau \geq 0
$$

and using (5.14) and (2.1), (5.16) assumes the form

$$
\begin{aligned}
W(\sigma(t), P)= & \frac{1}{2}\left\{\frac{c}{\Theta_{0}} \vartheta^{2}(t+d)+\frac{1}{\varepsilon}[\varepsilon \mathbf{E}(t+d)+\vartheta(t+d) \mathbf{a}]^{2}\right. \\
& \left.+\mu \mathbf{H}^{2}(t+d)\right\}-\frac{1}{2}\left\{\frac{c}{\Theta_{0}} \vartheta^{2}(t)+\frac{1}{\varepsilon}[\varepsilon \mathbf{E}(t)+\vartheta(t) \mathbf{a}]^{2}+\mu \mathbf{H}^{2}(t)\right\} \\
& +\frac{1}{2} \int_{0}^{+\infty} \int_{0}^{+\infty}\left[\alpha(|\tau-\xi|) \mathbf{E}_{P}(\xi) \cdot \mathbf{E}_{P}(\tau)\right. \\
& \left.\quad+\frac{1}{\Theta_{0}} k(|\tau-\xi|) \mathbf{g}_{P}(\xi) \cdot \mathbf{g}_{P}(\tau)\right] d \xi d \tau \\
& -\int_{0}^{+\infty} \mathbf{I}_{(\alpha)}\left(\tau, \mathbf{E}^{t}\right) \cdot \mathbf{E}_{P}(\tau) d \tau-\frac{1}{\Theta_{0}} \int_{0}^{+\infty} \mathbf{I}_{(k)}\left(\tau, \mathbf{g}^{t}\right) \cdot \mathbf{g}_{P}(\tau) d \tau,
\end{aligned}
$$


or the equivalent one

$$
\begin{aligned}
W(\sigma(t), P)= & \frac{1}{2}\left\{\frac{c}{\Theta_{0}} \vartheta^{2}(t+d)+\frac{1}{\varepsilon}[\varepsilon \mathbf{E}(t+d)+\vartheta(t+d) \mathbf{a}]^{2}\right. \\
& \left.+\mu \mathbf{H}^{2}(t+d)\right\}-\frac{1}{2}\left\{\frac{c}{\Theta_{0}} \vartheta^{2}(t)+\frac{1}{\varepsilon}[\varepsilon \mathbf{E}(t)+\vartheta(t) \mathbf{a}]^{2}+\mu \mathbf{H}^{2}(t)\right\} \\
& +\frac{1}{2 \pi} \int_{-\infty}^{+\infty}\left[\alpha_{c}(\omega) \mathbf{E}_{P+}(\omega) \cdot \mathbf{E}_{P+}^{*}(\omega) d \omega+\frac{1}{\Theta_{0}} k_{c}(\omega) \mathbf{g}_{P+}(\omega) \cdot \mathbf{g}_{P+}^{*}(\omega)\right] d \omega \\
& -\frac{1}{2 \pi} \int_{-\infty}^{+\infty}\left[\mathbf{I}_{(\alpha)+}\left(\omega, \mathbf{E}^{t}\right) \cdot \mathbf{E}_{P+}^{*}(\omega)+\frac{1}{\Theta_{0}} \mathbf{I}_{(k)+}\left(\omega, \mathbf{g}^{t}\right) \cdot \mathbf{g}_{P+}^{*}(\omega)\right] d \omega,
\end{aligned}
$$

where, on account of $(3.6)_{1}$, we have put

$$
\mathbf{I}_{(\alpha)+}\left(\omega, \mathbf{E}^{t}\right)=\int_{0}^{+\infty} \mathbf{I}_{(\alpha)}\left(\tau, \mathbf{E}^{t}\right) e^{-i \omega \tau} d \tau, \mathbf{I}_{(k)+}\left(\omega, \mathbf{g}^{t}\right)=\int_{0}^{+\infty} \mathbf{I}_{(k)}\left(\tau, \mathbf{g}^{t}\right) e^{-i \omega \tau} d \tau
$$

We note that the two functions $\mathbf{I}_{(\alpha)}\left(\tau, \mathbf{E}^{t}\right)$ and $\mathbf{I}_{(k)}\left(\tau, \mathbf{g}^{t}\right)$, defined by (5.17), are related to the electric current density and the heat flux corresponding to the static continuations of $\mathbf{E}^{t}$ and $\mathbf{g}^{t}$, already introduced with (3.1). In fact, when these static continuations are considered, we have the expressions (3.3) and (3.4), which, if the duration of the continuations is equal to $\tau$, reduce to

$$
\mathbf{J}\left(\mathbf{E}^{t_{(\tau)}}\right)-\nu^{(\alpha)}(\tau) \mathbf{E}(t)=-\mathbf{I}_{(\alpha)}\left(\tau, \mathbf{E}^{t}\right), \mathbf{q}\left(\mathbf{g}^{t_{(\tau)}}\right)-\nu^{(k)}(\tau) \mathbf{g}(t)=-\mathbf{I}_{(k)}\left(\tau, \mathbf{g}^{t}\right) .
$$

Hence, it follows that $\mathbf{I}_{(\alpha)}\left(\tau, \mathbf{E}^{t}\right)$ and $\mathbf{I}_{(k)}\left(\tau, \mathbf{g}^{t}\right)$ have the regularities of the quantities at the left hand-sides of (5.21).

The last expression of $W(\sigma(t), P)$, given by (5.19), allows us to state that the admissible histories $\mathbf{E}^{t}$ and $\mathbf{g}^{t}$ are those such that $\mathbf{I}_{(\alpha)}\left(\cdot, \mathbf{E}^{t}\right)$ and $\mathbf{I}_{(k)}\left(\cdot, \mathbf{g}^{t}\right)$, related to $\mathbf{E}^{t}$ and $\mathbf{g}^{t}$ by means of (5.17), belong to $H_{\alpha}^{\prime}\left(\mathbf{R}^{+}, \mathbf{R}^{3}\right)$ and $H_{k}^{\prime}\left(\mathbf{R}^{+}, \mathbf{R}^{3}\right)$, the dual spaces of $H_{\alpha}\left(\mathbf{R}^{+}, \mathbf{R}^{3}\right)$ and $H_{k}\left(\mathbf{R}^{+}, \mathbf{R}^{3}\right)$, whose functions satisfy

$$
|\langle\mathbf{f}, \mathbf{g}\rangle|=\left|\int_{0}^{+\infty} \mathbf{f}(t) \cdot \mathbf{g}(t) d t\right|=\frac{1}{2 \pi}\left|\int_{-\infty}^{+\infty} \mathbf{f}_{+}(\omega) \cdot \mathbf{g}_{+}^{*}(\omega) d \omega\right|<+\infty
$$

6. Equivalence of histories related to the work. Two couples of histories $\left(\mathbf{E}_{1}(t)\right.$, $\left.\mathbf{E}_{1}^{t}\right)$ with $\mathbf{g}_{1}^{t}$ and $\left(\mathbf{E}_{2}(t), \mathbf{E}_{2}^{t}\right)$ with $\mathbf{g}_{2}^{t}$, in Sec. 4 , have been called equivalent when they yield both the same electric current density and the same heat flux if they are subjected to the same process.

An analogous equivalence relation can be introduced by means of the thermoelectromagnetic work considered in the previous section. Thus, we give the following definition in terms of work.

Definition 6.1. Let $\left(\mathbf{E}_{j}(t), \mathbf{E}_{j}^{t}\right), j=1,2$, be two histories of the electric field and let $\mathbf{g}_{j}^{t}, j=1,2$, be two corresponding past histories of the temperature gradient, related to the instantaneous values $\mathbf{H}_{1}(t) \underset{\sim}{\mathbf{H}}(t)$ of the magnetic field and $\vartheta_{1}(t), \vartheta_{2}(t)$ of the temperature, respectively. They are called w-equivalent if for every $\dot{\mathbf{E}}_{P}:[0, \tau) \rightarrow \mathbf{R}^{3}$, 
$\dot{\mathbf{H}}_{P}:[0, \tau) \rightarrow \mathbf{R}^{3}, \dot{\vartheta}_{P}:[0, \tau) \rightarrow \mathbf{R}, \mathbf{g}_{P}:[0, \tau) \rightarrow \mathbf{R}^{3}$ and for every $\tau>0$, the equality

$$
\begin{aligned}
\tilde{W}\left(\mathbf{E}_{1}(t), \mathbf{H}_{1}(t), \vartheta_{1}(t), \mathbf{E}_{1}^{t}, \mathbf{g}_{1}^{t} ; \dot{\mathbf{E}}_{P}, \dot{\mathbf{H}}_{P}, \dot{\vartheta}_{P}, \mathbf{g}_{P}\right) \\
=\tilde{W}\left(\mathbf{E}_{2}(t), \mathbf{H}_{2}(t), \vartheta_{2}(t), \mathbf{E}_{2}^{t}, \mathbf{g}_{2}^{t} ; \dot{\mathbf{E}}_{P}, \dot{\mathbf{H}}_{P}, \dot{\vartheta}_{P}, \mathbf{g}_{P}\right)
\end{aligned}
$$

is satisfied.

The equivalence expressed by Definition 4.1 coincides with the one now introduced in the sense of the following theorem.

TheOREm 6.1. For every thermoelectromagnetic material $\mathcal{B}$, described by the constitutive equations (2.1) and (4.10), two couples of histories of the electric field and of the temperature gradient are w-equivalent if and only if they are equivalent in the sense of Definition 4.1.

Proof. If two couples of histories, $\left(\mathbf{E}_{j}(t), \mathbf{E}_{j}^{t}\right)$ with $\mathbf{g}_{j}^{t}, j=1,2$, satisfy Definition 4.1, then they are w-equivalent, too. In fact, for every $\mathbf{E}_{P}:(0, \tau] \rightarrow \mathbf{R}^{3}, \mathbf{H}_{P}:(0, \tau] \rightarrow \mathbf{R}^{3}$, $\vartheta_{P}:(0, \tau] \rightarrow \mathbf{R}, \mathbf{g}_{P}:(0, \tau] \rightarrow \mathbf{R}^{3}$ and for every $\tau>0$, from (5.1) we get

$$
\begin{aligned}
\int_{0}^{d}\left[\frac{c}{\Theta_{0}} \dot{\vartheta}_{P}(\tau) \vartheta_{P}(\tau)+\frac{1}{\varepsilon} \dot{\mathbf{D}}\left(\mathbf{E}_{P}(\tau), \vartheta_{P}(\tau)\right) \cdot \mathbf{D}\left(\mathbf{E}_{P}(\tau), \vartheta_{P}(\tau)\right)+\frac{1}{\mu} \dot{\mathbf{B}}\left(\mathbf{H}_{P}(\tau)\right)\right. \\
\left.\cdot \cdot \mathbf{B}\left(\mathbf{H}_{P}(\tau)\right)+\tilde{\mathbf{J}}\left(\left(\mathbf{E}_{P} * \mathbf{E}_{1}\right)^{t+\tau}\right) \cdot \mathbf{E}_{P}(\tau)-\frac{1}{\Theta_{0}} \tilde{\mathbf{q}}\left(\left(\mathbf{g}_{P} * \mathbf{g}_{1}\right)^{t+\tau}\right) \cdot \mathbf{g}_{P}(\tau)\right] d \tau \\
=\int_{0}^{d}\left[\frac{c}{\Theta_{0}} \dot{\vartheta}_{P}(\tau) \vartheta_{P}(\tau)+\frac{1}{\varepsilon} \dot{\mathbf{D}}\left(\mathbf{E}_{P}(\tau), \vartheta_{P}(\tau)\right) \cdot \mathbf{D}\left(\mathbf{E}_{P}(\tau), \vartheta_{P}(\tau)\right)+\frac{1}{\mu} \dot{\mathbf{B}}\left(\mathbf{H}_{P}(\tau)\right)\right. \\
\left.\cdot \mathbf{B}\left(\mathbf{H}_{P}(\tau)\right)+\tilde{\mathbf{J}}\left(\left(\mathbf{E}_{P} * \mathbf{E}_{2}\right)^{t+\tau}\right) \cdot \mathbf{E}_{P}(\tau)-\frac{1}{\Theta_{0}} \tilde{\mathbf{q}}\left(\left(\mathbf{g}_{P} * \mathbf{g}_{2}\right)^{t+\tau}\right) \cdot \mathbf{g}_{P}(\tau)\right] d \tau
\end{aligned}
$$

This relation expresses that the work done on the process starting from the state $\left(\mathbf{E}_{1}(t)\right.$, $\left.\mathbf{H}_{1}(t), \vartheta_{1}(t), \mathbf{E}_{1}^{t}, \mathbf{g}_{1}^{t}\right)$ coincides with the work done when the same process starts from $\left(\mathbf{E}_{2}(t), \mathbf{H}_{2}(t), \vartheta_{2}(t), \mathbf{E}_{2}^{t}, \mathbf{g}_{2}^{t}\right)$, since, by hypothesis, the instantaneous values present in the two states coincide as well as (4.11) $)_{2}$ and (4.12).

Now, if two couples of histories, $\left(\mathbf{E}_{j}(t), \mathbf{E}_{j}^{t}\right)$ with $\mathbf{g}_{j}^{t}, j=1,2$, are w-equivalent, then for any process $P=\left(\dot{\mathbf{E}}_{P}, \dot{\mathbf{H}}_{P}, \dot{\vartheta}_{P}, \mathbf{g}_{P}\right)$ and for every $d>0, d$ being the duration of the process, (6.1) must be satisfied. This equality, taking into account (5.16) or (5.18), which is equivalent because of (5.14) and (5.17), can be put in the following form:

$$
\begin{aligned}
\frac{c}{2 \Theta_{0}}\{ & \left\{\vartheta_{P_{1}}^{2}(d)-\vartheta_{P_{2}}^{2}(d)-\left[\vartheta_{P_{1}}^{2}(0)-\vartheta_{P_{2}}^{2}(0)\right]\right\}+\frac{1}{2 \varepsilon}\left\{\mathbf{D}_{P_{1}}^{2}(d)-\mathbf{D}_{P_{2}}^{2}(d)\right. \\
& \left.-\left[\mathbf{D}_{P_{1}}^{2}(0)-\mathbf{D}_{P_{2}}^{2}(0)\right]\right\}+\frac{1}{2 \mu}\left\{\mathbf{B}_{P_{1}}^{2}(d)-\mathbf{B}_{P_{2}}^{2}(d)-\left[\mathbf{B}_{P_{1}}^{2}(0)-\mathbf{B}_{P_{2}}^{2}(0)\right]\right\} \\
& +\frac{1}{2} \int_{0}^{+\infty} \int_{0}^{+\infty} \alpha(|\tau-\xi|)\left[\mathbf{E}_{P_{1}}(\xi) \cdot \mathbf{E}_{P_{1}}(\tau)-\mathbf{E}_{P_{2}}(\xi) \cdot \mathbf{E}_{P_{2}}(\tau)\right] d \xi d \tau \\
& -\int_{0}^{+\infty}\left[\mathbf{I}_{(\alpha)}\left(\tau, \mathbf{E}_{1}^{t}\right) \cdot \mathbf{E}_{P_{1}}(\tau)-\mathbf{I}_{(\alpha)}\left(\tau, \mathbf{E}_{2}^{t}\right) \cdot \mathbf{E}_{P_{2}}(\tau)\right] d \tau \\
& -\frac{1}{\Theta_{0}} \int_{0}^{+\infty}\left[\mathbf{I}_{(k)}\left(\tau, \mathbf{g}_{1}^{t}\right)-\mathbf{I}_{(k)}\left(\tau, \mathbf{g}_{2}^{t}\right)\right] \cdot \mathbf{g}_{P}(\tau) d \tau=0 .
\end{aligned}
$$


Here, for example, $\vartheta_{P_{i}}(0)=\vartheta_{i}(t)$ and $\vartheta_{P_{i}}(d)=\vartheta_{i}(t)+\int_{0}^{d} \dot{\vartheta}_{P}(s) d s$ with $i=1,2$ is due to (4.6), while $\mathbf{E}_{P_{i}}$ and $\mathbf{H}_{P_{i}}$ have analogous expressions by virtue of (4.4) and (4.5). Moreover, $\mathbf{D}_{P_{i}}$ is expressed in terms of the corresponding $\mathbf{E}_{P_{i}}$ and $\vartheta_{P_{i}}$ taking into account (2.1), as well as $\mathbf{B}_{P_{i}}$ in function of $\mathbf{H}_{P_{i}}$ and, in particular, obviously $\mathbf{E}_{P_{i}}(\xi)=\mathbf{E}_{i}(t)+\int_{0}^{\xi}$ $\dot{\mathbf{E}}_{P}(s) d s$. We note that in (6.3) the integral with $k(|\tau-\xi|)$ has been eliminated since we have the presence of the same $\mathbf{g}_{P}$ on both sides of (6.1).

Thus, (6.3) can be written as follows:

$$
\begin{aligned}
& \left\{\left(\frac{c}{\Theta_{0}}+\frac{\mathbf{a}^{2}}{\varepsilon}\right)\left[\vartheta_{1}(t)-\vartheta_{2}(t)\right]+\mathbf{a} \cdot\left[\mathbf{E}_{1}(t)-\mathbf{E}_{2}(t)\right]\right\} \int_{0}^{d} \dot{\vartheta}_{P}(s) d s \\
& \quad+\left\{\mathbf{a}\left[\vartheta_{1}(t)-\vartheta_{2}(t)\right]+\varepsilon\left[\mathbf{E}_{1}(t)-\mathbf{E}_{2}(t)\right]\right\} \cdot \int_{0}^{d} \dot{\mathbf{E}}_{P}(s) d s+\mu\left[\mathbf{H}_{1}(t)\right. \\
& \left.\quad-\mathbf{H}_{2}(t)\right] \cdot \int_{0}^{d} \dot{\mathbf{H}}_{P}(s) d s+\frac{1}{2}\left\{\left[\mathbf{E}_{1}^{2}(t)-\mathbf{E}_{2}^{2}(t)\right] \int_{0}^{+\infty} \int_{0}^{+\infty} \alpha(|\tau-\xi|) d \xi d \tau\right. \\
& \left.\quad+\left[\mathbf{E}_{1}(t)-\mathbf{E}_{2}(t)\right] \cdot \int_{0}^{+\infty} \int_{0}^{+\infty} \alpha(|\tau-\xi|)\left[\int_{0}^{\xi} \dot{\mathbf{E}}_{P}(s) d s+\int_{0}^{\tau} \dot{\mathbf{E}}_{P}(s) d s\right] d \xi d \tau\right\} \\
& \quad-\int_{0}^{+\infty}\left[\mathbf{I}_{(\alpha)}\left(\tau, \mathbf{E}_{1}^{t}\right) \cdot \mathbf{E}_{1}(t)-\mathbf{I}_{(\alpha)}\left(\tau, \mathbf{E}_{2}^{t}\right) \cdot \mathbf{E}_{2}(t)\right] d \tau-\int_{0}^{+\infty}\left[\mathbf{I}_{(\alpha)}\left(\tau, \mathbf{E}_{1}^{t}\right)-\mathbf{I}_{(\alpha)}\left(\tau, \mathbf{E}_{2}^{t}\right)\right] \\
& \quad \cdot\left[\int_{0}^{\tau} \dot{\mathbf{E}}_{P}(s) d s\right] d \tau-\frac{1}{\Theta_{0}} \int_{0}^{+\infty}\left[\mathbf{I}_{(k)}\left(\tau, \mathbf{g}_{1}^{t}\right)-\mathbf{I}_{(k)}\left(\tau, \mathbf{g}_{2}^{t}\right)\right] \cdot \mathbf{g}_{P}(\tau) d \tau=0 .
\end{aligned}
$$

This relation must be satisfied by any process $P$ and any $d>0$. Thus, we first observe that the arbitrarinesses of $\dot{\vartheta}_{P}$ and $\dot{\mathbf{E}}_{P}$ imply that the first two bracketed quantities must vanish, i.e.,

$$
\left\{\begin{array}{l}
\left(\frac{c}{\Theta_{0}}+\frac{\mathbf{a}^{2}}{\varepsilon}\right)\left[\vartheta_{1}(t)-\vartheta_{2}(t)\right]+\mathbf{a} \cdot\left[\mathbf{E}_{1}(t)-\mathbf{E}_{2}(t)\right]=0, \\
\mathbf{a}\left[\vartheta_{1}(t)-\vartheta_{2}(t)\right]+\varepsilon\left[\mathbf{E}_{1}(t)-\mathbf{E}_{2}(t)\right]=\mathbf{0} .
\end{array}\right.
$$

Hence, substituting into the first equation the expression of $\mathbf{E}_{1}(t)-\mathbf{E}_{2}(t)$ obtained from the second one, it follows that

$$
\vartheta_{1}(t)=\vartheta_{2}(t), \quad \mathbf{E}_{1}(t)=\mathbf{E}_{2}(t) .
$$

Moreover, also being $\dot{\mathbf{H}}_{P}$ arbitrary, we get

$$
\mathbf{H}_{1}(t)=\mathbf{H}_{2}(t)
$$

furthermore, for the arbitrariness of $\mathbf{g}_{P}$ too, we must have

$$
\mathbf{I}_{(\alpha)}\left(\tau, \mathbf{E}_{1}^{t}\right)=\mathbf{I}_{(\alpha)}\left(\tau, \mathbf{E}_{2}^{t}\right), \quad \mathbf{I}_{(k)}\left(\tau, \mathbf{g}_{1}^{t}\right)=\mathbf{I}_{(k)}\left(\tau, \mathbf{g}_{2}^{t}\right) .
$$

The last two equalities, by virtue of (5.17), yield

$$
\int_{0}^{+\infty} \alpha(\tau+\eta)\left[\mathbf{E}_{1}^{t}(\eta)-\mathbf{E}_{2}^{t}(\eta)\right] d \eta=\mathbf{0}, \int_{0}^{+\infty} k(\tau+\eta)\left[\mathbf{g}_{1}^{t}(\eta)-\mathbf{g}_{2}^{t}(\eta)\right] d \eta, \forall \tau>0,
$$

from which, taking into account $(6.6)_{2}$, it follows that the couple of histories $\left(\mathbf{0}, \mathbf{E}_{1}^{t}-\mathbf{E}_{2}^{t}\right)$ and $\mathbf{g}_{1}^{t}-\mathbf{g}_{2}^{t}$ is equivalent to the null histories $\left(\mathbf{0}, \mathbf{0}^{\dagger}\right)$ and $\mathbf{0}^{\dagger}$. Thus, the two couples of histories $\left(\mathbf{E}_{j}(t), \mathbf{E}_{j}^{t}\right)$ and $\mathbf{g}_{j}^{t}, j=1,2$, are equivalent in the sense of Definition 4.1. 
7. Minimum free energy. The maximum recoverable work that we can obtain starting from a given state $\sigma$ of the body indicates the maximum quantity of work we can get from the material at the given state $\sigma$. It is related to the amount of the available energy and is defined by 12 .

$$
W_{R}(\sigma)=\sup \{-W(\sigma, P): P \in \Pi\},
$$

where $\Pi$ denotes the set of finite work processes.

We observe that $W_{R}(\sigma)$ is a function of the state, which is non-negative since the null process belongs to $\Pi$ and the corresponding work is null. Moreover, on the ground of thermodynamic considerations, it is bounded above, i.e. $W_{R}(\sigma)<+\infty$.

As many authors have already shown [3, 5, 12, denoting by $\psi_{m}(\sigma)$ the minimum free energy, we have

$$
\psi_{m}(\sigma)=W_{R}(\sigma) .
$$

Let us consider a process $P \in \Pi$, which is related to $\mathbf{E}_{P}, \mathbf{H}_{P}$ and $\vartheta_{P}$, whose expressions are given by (4.4)-(4.6) and defined on $(0, d], d$ being the duration of $P$. Putting $P=0$ on $[d,+\infty)$, we can extend $P$ on $\mathbf{R}^{+}$; moreover, we suppose that $\mathbf{E}_{P}(d) \equiv \mathbf{E}(t+d)=\mathbf{0}$, $\mathbf{H}_{P}(d) \equiv \mathbf{H}(t+d)=\mathbf{0}, \vartheta_{P}(d) \equiv \vartheta(t+d)=0$. The work done on $P$, starting from the state $\sigma(t)=\left(\mathbf{E}(t), \mathbf{H}(t), \vartheta(t), \mathbf{E}^{t}, \mathbf{g}^{t}\right)$ where $t$ is a fixed time instant, is given by (5.18), which reduces to

$$
\begin{aligned}
W(\sigma(t), P) & \\
& =-\frac{1}{2}\left\{\frac{c}{\Theta_{0}} \vartheta^{2}(t)+\frac{1}{\varepsilon}[\varepsilon \mathbf{E}(t)+\vartheta(t) \mathbf{a}]^{2}+\mu \mathbf{H}^{2}(t)\right\} \\
& +\frac{1}{2} \int_{0}^{+\infty} \int_{0}^{+\infty}\left[\alpha(|\tau-\xi|) \mathbf{E}_{P}(\xi) \cdot \mathbf{E}_{P}(\tau)+\frac{1}{\Theta_{0}} k(|\tau-\xi|) \mathbf{g}_{P}(\xi) \cdot \mathbf{g}_{P}(\tau)\right] d \xi d \tau \\
& -\int_{0}^{+\infty}\left[\mathbf{I}_{(\alpha)}\left(\tau, \mathbf{E}^{t}\right) \cdot \mathbf{E}_{P}(\tau)+\frac{1}{\Theta_{0}} \mathbf{I}_{(k)}\left(\tau, \mathbf{g}^{t}\right) \cdot \mathbf{g}_{P}(\tau)\right] d \tau .
\end{aligned}
$$

We must derive the maximum recoverable work, which will correspond to an "optimal" process $P^{(m)}$. Denoting by $\mathbf{E}^{(m)}$ and $\mathbf{g}^{(m)}$ the required solutions, related to $P^{(m)}$, we must derive the maximum of $-W(\sigma, P)$, due to (7.1). To this purpose, in correspondence of any process $P$, we put

$$
\mathbf{E}_{P}(\tau)=\mathbf{E}^{(m)}(\tau)+\gamma \mathbf{e}(\tau), \quad \mathbf{g}_{P}(\tau)=\mathbf{g}^{(m)}(\tau)+\delta \mathbf{v}(\tau), \quad \tau \in \mathbf{R}^{+},
$$

where $\gamma$ and $\delta$ are two real parameters, and $\mathbf{e}$ and $\mathbf{v}$ are two arbitrary smooth functions such that $\mathbf{e}(0)=\mathbf{0}$ and $\mathbf{v}(0)=\mathbf{0}$.

From (7.3), after substituting (7.4), we get the following system:

$$
\left\{\begin{array}{l}
\left.\frac{\partial}{\partial \gamma}[-W(\sigma, P)]\right|_{\gamma=0}=-\int_{0}^{+\infty}\left[\int_{0}^{+\infty} \alpha(|\tau-\xi|) \mathbf{E}^{(m)}(\xi) d \xi-\mathbf{I}_{(\alpha)}\left(\tau, \mathbf{E}^{t}\right)\right] \cdot \mathbf{e}(\tau) d \tau=0, \\
\left.\frac{\partial}{\partial \delta}[-W(\sigma, P)]\right|_{\delta=0}=-\frac{1}{\Theta_{0}} \int_{0}^{+\infty}\left[\int_{0}^{+\infty} k(|\tau-\xi|) \mathbf{g}^{(m)}(\xi) d \xi-\mathbf{I}_{(k)}\left(\tau, \mathbf{g}^{t}\right)\right] \cdot \mathbf{v}(\tau) d \tau=0,
\end{array}\right.
$$

from which, e and $\mathbf{v}$ being arbitrary, it follows that

$$
\begin{cases}\int_{0}^{+\infty} \alpha(|\tau-\xi|) \mathbf{E}^{(m)}(\xi) d \xi=\mathbf{I}_{(\alpha)}\left(\tau, \mathbf{E}^{t}\right), & \forall \tau \in \mathbf{R}^{+} \\ \int_{0}^{+\infty} k(|\tau-\xi|) \mathbf{g}^{(m)}(\xi) d \xi=\mathbf{I}_{(k)}\left(\tau, \mathbf{g}^{t}\right), & \forall \tau \in \mathbf{R}^{+}\end{cases}
$$


These last two relations are two integral equations of the Wiener-Hopf type, of the first kind, whose solutions $\mathbf{E}^{(m)}$ and $\mathbf{g}^{(m)}$ can be determined by virtue of the thermodynamic properties of the two kernels $\alpha$ and $k$, together with some theorems on factorization.

Thus, these solutions allow us to obtain the minimum free energy (7.2), which, taking into account (7.3) and (7.6), becomes

$$
\begin{aligned}
\psi_{m}(\sigma)= & W_{R}(\sigma)=\frac{1}{2}\left\{\frac{c}{\Theta_{0}} \vartheta^{2}(t)+\frac{1}{\varepsilon}[\varepsilon \mathbf{E}(t)+\vartheta(t) \mathbf{a}]^{2}+\mu \mathbf{H}^{2}(t)\right\} \\
& +\frac{1}{2} \int_{0}^{+\infty} \int_{0}^{+\infty}\left[\alpha(|\tau-\xi|) \mathbf{E}^{(m)}(\xi) \cdot \mathbf{E}^{(m)}(\tau)\right. \\
& \left.+\frac{1}{\Theta_{0}} k(|\tau-\xi|) \mathbf{g}^{(m)}(\xi) \cdot \mathbf{g}^{(m)}(\tau)\right] d \xi d \tau \\
= & \frac{1}{2}\left\{\frac{c}{\Theta_{0}} \vartheta^{2}(t)+\frac{1}{\varepsilon}[\varepsilon \mathbf{E}(t)+\vartheta(t) \mathbf{a}]^{2}+\mu \mathbf{H}^{2}(t)\right\} \\
& +\frac{1}{2 \pi} \int_{-\infty}^{+\infty}\left[\alpha_{c}(\omega) \mathbf{E}_{+}^{(m)}(\omega) \cdot\left(\mathbf{E}_{+}^{(m)}(\omega)\right)^{*}+\frac{1}{\Theta_{0}} k_{c}(\omega) \mathbf{g}_{+}^{(m)}(\omega) \cdot\left(\mathbf{g}_{+}^{(m)}(\omega)\right)^{*}\right] d \omega
\end{aligned}
$$

where we have applied Plancherel's theorem.

To solve the Wiener-Hopf integral equations (7.6) of the first kind, we transform them as follows:

$$
\begin{cases}\int_{0}^{+\infty} \alpha(|\tau-\xi|) \mathbf{E}^{(m)}(\xi) d \xi=\mathbf{I}_{(\alpha)}\left(\tau, \mathbf{E}^{t}\right)+\mathbf{r}^{(\alpha)}(\tau), & \forall \tau \in \mathbf{R} \\ \int_{0}^{+\infty} k(|\tau-\xi|) \mathbf{g}^{(m)}(\xi) d \xi=\mathbf{I}_{(k)}\left(\tau, \mathbf{g}^{t}\right)+\mathbf{r}^{(k)}(\tau), & \forall \tau \in \mathbf{R}\end{cases}
$$

where we have introduced

$$
\mathbf{r}^{(\alpha)}(\tau)=\int_{-\infty}^{+\infty} \alpha(|\tau-\xi|) \mathbf{E}^{(m)}(\xi) d \xi, \mathbf{r}^{(k)}(\tau)=\int_{-\infty}^{+\infty} k(|\tau-\xi|) \mathbf{g}^{(m)}(\xi) d \xi \quad \forall \tau \in \mathbf{R}^{-}
$$

with $\mathbf{r}^{(\alpha)}(\tau)=\mathbf{0}, \mathbf{r}^{(k)}(\tau)=\mathbf{0} \forall \tau \in \mathbf{R}^{++}$, being $\operatorname{supp}\left(\mathbf{E}^{(m)}\right) \subseteq \mathbf{R}^{+}, \operatorname{supp}\left(\mathbf{g}^{(m)}\right) \subseteq \mathbf{R}^{+}$, $\operatorname{supp}\left(\mathbf{I}_{(\alpha)}\left(\cdot, \mathbf{E}^{t}\right)\right) \subseteq \mathbf{R}^{+}, \operatorname{supp}\left(\mathbf{I}_{(k)}\left(\cdot, \mathbf{g}^{t}\right)\right) \subseteq \mathbf{R}^{+}, \operatorname{supp}\left(\mathbf{r}^{(\alpha)}\right) \subseteq \mathbf{R}^{-}$and $\operatorname{supp}\left(\mathbf{r}^{(k)}\right) \subseteq \mathbf{R}^{-}$.

Thus, we can apply Fourier's transform to (7.8) and obtain

$$
2 \alpha_{c}(\omega) \mathbf{E}_{+}^{(m)}(\omega)=\mathbf{I}_{(\alpha)+}^{t}(\omega)+\mathbf{r}_{-}^{(\alpha)}(\omega), 2 k_{c}(\omega) \mathbf{g}_{+}^{(m)}(\omega)=\mathbf{I}_{(k)+}^{t}(\omega)+\mathbf{r}_{-}^{(k)}(\omega),
$$

where $\mathbf{I}_{(\alpha)+}^{t}(\omega)=\mathbf{I}_{(\alpha)+}\left(\omega, \mathbf{E}^{t}\right), \mathbf{I}_{(k)+}^{t}(\omega)=\mathbf{I}_{(k)+}\left(\omega, \mathbf{g}^{t}\right)$ given by (5.20).

In the complex $z$-plane $\mathbf{C}$, the upper half-planes including and excluding the real axis are denoted by

$$
\mathbf{C}^{+}=\left\{z \in \mathbf{C}: \operatorname{Im} z \in \mathbf{R}^{+}\right\}, \quad \mathbf{C}^{(+)}=\left\{z \in \mathbf{C}: \operatorname{Im} z \in \mathbf{R}^{++}\right\} .
$$

Similarly, we can define the lower half-planes $\mathbf{C}^{-}$and $\mathbf{C}^{(-)}$, respectively including and excluding the real axis. We note that the two functions $f_{ \pm}(z)$ defined by (3.6) are analytic for $z \in \mathbf{C}^{(\mp)}$, respectively. By assumption, the analyticities in $\mathbf{C}^{(\mp)}$ are extended to $\mathbf{C}^{\mp}$. It is possible to define, for example, $f_{+}$in all or part of $\mathbf{C}^{(+)}$by analytic continuation.

Thus, in particular, we note that $\mathbf{E}_{+}^{(m)}$ and $\mathbf{g}_{+}^{(m)}$ are analytic in $\mathbf{C}^{-}$.

Let us introduce the following two functions:

$$
K^{(\alpha)}(\omega)=\left(1+\omega^{2}\right) \alpha_{c}(\omega), \quad K^{(k)}(\omega)=\left(1+\omega^{2}\right) k_{c}(\omega) .
$$


They have no zero for any real $\omega$, also at infinity, by virtue of (3.15), (3.16) and the analogous relations, which hold for the kernel $\alpha$. Moreover, they can be factorized as follows:

$$
K^{(\alpha)}(\omega)=K_{(+)}^{(\alpha)}(\omega) K_{(-)}^{(\alpha)}(\omega), \quad K^{(k)}(\omega)=K_{(+)}^{(k)}(\omega) K_{(-)}^{(k)}(\omega),
$$

where henceforth the subscript $( \pm)$ indicates that the function $f(z)$ has zeros and singularities only for $z \in \mathbf{C}^{ \pm}$.

Also $\alpha_{c}(\omega)$ and $k_{c}(\omega)$ can be factorized as follows:

$$
\alpha_{c}(\omega)=\alpha_{(+)}(\omega) \alpha_{(-)}(\omega), \quad k_{c}(\omega)=k_{(+)}(\omega) k_{(-)}(\omega) .
$$

Therefore, from (7.11) and (7.12) we obtain

$$
\alpha_{( \pm)}(\omega)=\frac{1}{1 \pm i \omega} K_{( \pm)}^{(\alpha)}(\omega), \quad k_{( \pm)}(\omega)=\frac{1}{1 \pm i \omega} K_{( \pm)}^{(k)}(\omega),
$$

which allow us to get from (7.10)

$$
\begin{aligned}
\alpha_{(+)}(\omega) \mathbf{E}_{+}^{(m)}(\omega) & =\frac{1}{2 \alpha_{(-)}(\omega)}\left[\mathbf{I}_{(\alpha)+}^{t}(\omega)+\mathbf{r}_{-}^{(\alpha)}(\omega)\right], \\
k_{(+)}(\omega) \mathbf{g}_{+}^{(m)}(\omega) & =\frac{1}{2 k_{(-)}(\omega)}\left[\mathbf{I}_{(k)+}^{t}(\omega)+\mathbf{r}_{-}^{(k)}(\omega)\right] .
\end{aligned}
$$

Let $\mathbf{p}_{(\alpha)( \pm)}^{t}(\omega)$ and $\mathbf{p}_{(k)( \pm)}^{t}(\omega)$ be two functions defined by

$$
\begin{aligned}
& \mathbf{p}_{(\alpha)}^{t}(z)=\frac{1}{4 \pi i} \int_{-\infty}^{+\infty} \frac{\mathbf{I}_{(\alpha)+}^{t}(\omega) / \alpha_{(-)}(\omega)}{\omega-z} d \omega, \mathbf{p}_{(\alpha)( \pm)}^{t}(\omega)=\lim _{\beta \rightarrow 0 \mp} \mathbf{p}_{(\alpha)}^{t}(\omega+i \beta), \\
& \mathbf{p}_{(k)}^{t}(z)=\frac{1}{4 \pi i} \int_{-\infty}^{+\infty} \frac{\mathbf{I}_{(k)+}^{t}(\omega) / k_{(-)}(\omega)}{\omega-z} d \omega, \mathbf{p}_{(k)( \pm)}^{t}(\omega)=\lim _{\beta \rightarrow 0 \mp} \mathbf{p}_{(k)}^{t}(\omega+i \beta) .
\end{aligned}
$$

Then, using the Plemelj formulae 23] it follows that

$$
\begin{aligned}
& \frac{\mathbf{I}_{(\alpha)+}^{t}(\omega)}{2 \alpha_{(-)}(\omega)}=\mathbf{p}_{(\alpha)(-)}^{t}(\omega)-\mathbf{p}_{(\alpha)(+)}^{t}(\omega), \\
& \frac{\mathbf{I}_{(k)+}^{t}(\omega)}{2 k_{(-)}(\omega)}=\mathbf{p}_{(k)(-)}^{t}(\omega)-\mathbf{p}_{(k)(+)}^{t}(\omega) .
\end{aligned}
$$

We note that, since $\mathbf{p}_{(\alpha)( \pm)}^{t}(z)$ and $\mathbf{p}_{(k)( \pm)}^{t}(z)$ have zeros and singularities in $z \in \mathbf{C}^{ \pm}$, it follows that $\mathbf{p}_{(\alpha)(+)}^{t}(z)$ and $\mathbf{p}_{(k)(+)}^{t}(z)$ are analytic in $\mathbf{C}^{(-)}$, while $\mathbf{p}_{(\alpha)(-)}^{t}(z)$ and $\mathbf{p}_{(k)(-)}^{t}(z)$ are analytic in $\mathbf{C}^{(+)}$. Furthermore, for the assumption on the analyticity on the real axis of Fourier-transformed functions 3, all these functions are analytic on $\mathbf{R}$.

Thus, substituting (7.19) and (7.20) into (7.15) and (7.16), respectively, we obtain the following two relations:

$$
\begin{aligned}
\alpha_{(+)}(\omega) \mathbf{E}_{+}^{(m)}(\omega)+\mathbf{p}_{(\alpha)(+)}^{t}(\omega) & =\mathbf{p}_{(\alpha)(-)}^{t}(\omega)+\frac{1}{2} \frac{\mathbf{r}_{-}^{(\alpha)}(\omega)}{\alpha_{(-)}(\omega)}, \\
k_{(+)}(\omega) \mathbf{g}_{+}^{(m)}(\omega)+\mathbf{p}_{(k)(+)}^{t}(\omega) & =\mathbf{p}_{(k)(-)}^{t}(\omega)+\frac{1}{2} \frac{\mathbf{r}_{-}^{(k)}(\omega)}{k_{(-)}(\omega)}
\end{aligned}
$$


where the first left-hand sides are analytic for $z \in \mathbf{C}^{-}$, while the second right-hand sides are analytic for $z \in \mathbf{C}^{+}$. Hence, these relations define two functions, say

$$
\mathbf{M}_{\alpha}(\omega)=\alpha_{(+)}(\omega) \mathbf{E}_{+}^{(m)}(\omega)+\mathbf{p}_{(\alpha)(+)}^{t}(\omega), \mathbf{M}_{k}(\omega)=k_{(+)}(\omega) \mathbf{g}_{+}^{(m)}(\omega)+\mathbf{p}_{(k)(+)}^{t}(\omega),
$$

which are analytic on $\mathbf{C}^{-}$and at the same time on $\mathbf{C}^{+}$, by virtue of (7.21) and (7.22); therefore, they have analytic extensions on $\mathbf{C}$ and vanish at infinity. Hence, it follows that $\mathbf{M}_{\alpha}(\omega)=\mathbf{0}$ and $\mathbf{M}_{k}(\omega)=\mathbf{0}$; consequently, we get

$$
\begin{aligned}
& \mathbf{E}_{+}^{(m)}(\omega)=-\frac{\mathbf{p}_{(\alpha)(+)}^{t}(\omega)}{\alpha_{(+)}(\omega)}, \quad \mathbf{p}_{(\alpha)(-)}^{t}(\omega)=-\frac{1}{2} \frac{\mathbf{r}_{-}^{(\alpha)}(\omega)}{\alpha_{(-)}(\omega)}, \\
& \mathbf{g}_{+}^{(m)}(\omega)=-\frac{\mathbf{p}_{(k)(+)}^{t}(\omega)}{k_{(+)}(\omega)}, \quad \mathbf{p}_{(k)(-)}^{t}(\omega)=-\frac{1}{2} \frac{\mathbf{r}_{-}^{(k)}(\omega)}{k_{(-)}(\omega)}
\end{aligned}
$$

Finally, we obtain the expression of the minimum free energy from $(7.7)_{2}$, due to $(7.24)_{1}$ and $(7.25)_{1}$, that is,

$$
\begin{aligned}
\psi_{m}(\sigma(t))= & \frac{1}{2}\left\{\frac{c}{\Theta_{0}} \vartheta^{2}(t)+\frac{1}{\varepsilon}[\varepsilon \mathbf{E}(t)+\vartheta(t) \mathbf{a}]^{2}+\mu \mathbf{H}^{2}(t)\right\} \\
& +\frac{1}{2 \pi} \int_{-\infty}^{+\infty}\left[\left|\mathbf{p}_{(\alpha)(+)}^{t}(\omega)\right|^{2}+\frac{1}{\Theta_{0}}\left|\mathbf{p}_{(k)(+)}^{t}(\omega)\right|^{2}\right] d \omega .
\end{aligned}
$$

8. A different expression of the minimum free energy. The relation (7.26) derived for the minimum free energy $\psi_{m}$ is a function of $\mathbf{p}_{(\alpha)(+)}^{t}(\omega)$ and $\mathbf{p}_{(k)(+)}^{t}(\omega)$. These two quantities can be expressed in terms of $\mathbf{E}^{t}$ and $\mathbf{g}^{t}$, respectively.

To derive these relations, let us consider the causal extensions to $\mathbf{R}^{--}$of $\mathbf{E}^{t}(s)$ and $\mathbf{g}^{t}(s)$, which are identified with them; therefore, we have $\mathbf{E}^{t}(s)=\mathbf{0}$ and $\mathbf{g}^{t}(s)=\mathbf{0}$ for every $s \in(-\infty, 0)$. Moreover, the kernels $\alpha(s)$ and $k(s)$ are assumed to be even functions and, therefore, are denoted by $\alpha^{(e)}(s)$ and $k^{(e)}(s)$, whose Fourier transforms are $\alpha_{F}^{(e)}(\omega)=2 \alpha_{c}(\omega)$ and $k_{F}^{(e)}(\omega)=2 k_{c}(\omega)$.

Thus, (5.17) can be rewritten as

$$
\begin{aligned}
& \mathbf{I}_{(\alpha)}\left(\tau, \mathbf{E}^{t}\right)=-\int_{-\infty}^{+\infty} \alpha^{(e)}(\eta+\tau) \mathbf{E}^{t}(\eta) d \eta, \quad \tau \geq 0, \\
& \mathbf{I}_{(k)}\left(\tau, \mathbf{g}^{t}\right)=-\int_{-\infty}^{+\infty} k^{(e)}(\eta+\tau) \mathbf{g}^{t}(\eta) d \eta, \quad \tau \geq 0 .
\end{aligned}
$$

Then, by introducing

$$
\begin{aligned}
& \mathbf{I}_{(\alpha)}^{(n)}\left(\tau, \mathbf{E}^{t}\right)=-\int_{-\infty}^{+\infty} \alpha^{(e)}(\eta+\tau) \mathbf{E}^{t}(\eta) d \eta, \quad \forall \tau<0, \\
& \mathbf{I}_{(k)}^{(n)}\left(\tau, \mathbf{g}^{t}\right)=-\int_{-\infty}^{+\infty} k^{(e)}(\eta+\tau) \mathbf{g}^{t}(\eta) d \eta, \quad \forall \tau<0,
\end{aligned}
$$


we can extend (8.1) and (8.2) to $\mathbf{R}$ as follows:

$$
\begin{aligned}
& \mathbf{I}_{(\alpha)}^{(\mathbf{R})}\left(\tau, \mathbf{E}^{t}\right)=-\int_{-\infty}^{+\infty} \alpha^{(e)}(\eta+\tau) \mathbf{E}^{t}(\eta) d \eta= \begin{cases}\mathbf{I}_{(\alpha)}\left(\tau, \mathbf{E}^{t}\right) & \forall \tau \geq 0, \\
\mathbf{I}_{(\alpha)}^{(n)}\left(\tau, \mathbf{E}^{t}\right) & \forall \tau<0,\end{cases} \\
& \mathbf{I}_{(k)}^{(\mathbf{R})}\left(\tau, \mathbf{g}^{t}\right)=-\int_{-\infty}^{+\infty} k^{(e)}(\eta+\tau) \mathbf{g}^{t}(\eta) d \eta= \begin{cases}\mathbf{I}_{(k)}\left(\tau, \mathbf{g}^{t}\right) & \forall \tau \geq 0, \\
\mathbf{I}_{(k)}^{(n)}\left(\tau, \mathbf{g}^{t}\right) & \forall \tau<0 .\end{cases}
\end{aligned}
$$

Now, we can express these relations in terms of Fourier's transforms, that is,

$$
\begin{aligned}
& \mathbf{I}_{(\alpha)_{F}}^{(\mathbf{R})}\left(\omega, \mathbf{E}^{t}\right)=\int_{-\infty}^{+\infty} \mathbf{I}_{(\alpha)}^{(\mathbf{R})}\left(\tau, \mathbf{E}^{t}\right) e^{-i \omega \tau} d \tau=\mathbf{I}_{(\alpha)-}^{(n)}\left(\omega, \mathbf{E}^{t}\right)+\mathbf{I}_{(\alpha)+}\left(\omega, \mathbf{E}^{t}\right), \\
& \mathbf{I}_{(k)_{F}}^{(\mathbf{R})}\left(\omega, \mathbf{g}^{t}\right)=\int_{-\infty}^{+\infty} \mathbf{I}_{(k)}^{(\mathbf{R})}\left(\tau, \mathbf{g}^{t}\right) e^{-i \omega \tau} d \tau=\mathbf{I}_{(k)-}^{(n)}\left(\omega, \mathbf{g}^{t}\right)+\mathbf{I}_{(k)+}\left(\omega, \mathbf{g}^{t}\right),
\end{aligned}
$$

so that, by virtue of (7.19) and (7.20), we get

$$
\begin{aligned}
\frac{1}{2 \alpha_{(-)}(\omega)} \mathbf{I}_{(\alpha)_{F}}^{(\mathbf{R})}\left(\omega, \mathbf{E}^{t}\right) & =\frac{1}{2 \alpha_{(-)}(\omega)} \mathbf{I}_{(\alpha)-}^{(n)}\left(\omega, \mathbf{E}^{t}\right)+\mathbf{p}_{(\alpha)(-)}^{t}(\omega)-\mathbf{p}_{(\alpha)(+)}^{t}(\omega), \\
\frac{1}{2 k_{(-)}(\omega)} \mathbf{I}_{(k)_{F}}^{(\mathbf{R})}\left(\omega, \mathbf{g}^{t}\right) & =\frac{1}{2 k_{(-)}(\omega)} \mathbf{I}_{(k)-}^{(n)}\left(\omega, \mathbf{g}^{t}\right)+\mathbf{p}_{(k)(-)}^{t}(\omega)-\mathbf{p}_{(k)(+)}^{t}(\omega) .
\end{aligned}
$$

By using the Plemelj formulae, the quantities at the left-hand sides of the two last relations may be written as follows:

$$
\begin{aligned}
\frac{1}{2 \alpha_{(-)}(\omega)} \mathbf{I}_{(\alpha)_{F}}^{(\mathbf{R})}\left(\omega, \mathbf{E}^{t}\right) & =\mathbf{p}_{(\alpha)(-)}^{\prime t}(\omega)-\mathbf{p}_{(\alpha)(+)}^{\prime t}(\omega), \\
\frac{1}{2 k_{(-)}(\omega)} \mathbf{I}_{(k)_{F}}^{(\mathbf{R})}\left(\omega, \mathbf{g}^{t}\right) & =\mathbf{p}_{(k)(-)}^{\prime t}(\omega)-\mathbf{p}_{(k)(+)}^{\prime t}(\omega),
\end{aligned}
$$

where the functions $\mathbf{p}_{(\alpha)( \pm)}^{\prime t}(z)$ and $\mathbf{p}_{(k)( \pm)}^{\prime t}(z)$, defined analogously to (7.17) and (7.18), have zeros and singularities for $z \in \mathbf{C}^{ \pm}$.

Thus, (8.9) and (8.10), by virtue of (8.11) and (8.12), give two relations, which allow us to define the following two functions:

$$
\begin{aligned}
\mathbf{N}_{\alpha}(\omega) & \equiv \mathbf{p}_{(\alpha)(+)}^{t}(\omega)-\mathbf{p}_{(\alpha)(+)}^{\prime t}(\omega) \\
& =\mathbf{p}_{(\alpha)(-)}^{t}(\omega)-\mathbf{p}_{(\alpha)(-)}^{\prime t}(\omega)+\frac{1}{2 \alpha_{(-)}(\omega)} \mathbf{I}_{(\alpha)-}^{(n)}\left(\omega, \mathbf{E}^{t}\right) \\
\mathbf{N}_{k}(\omega) & \equiv \mathbf{p}_{(k)(+)}^{t}(\omega)-\mathbf{p}_{(k)(+)}^{\prime t}(\omega) \\
& =\mathbf{p}_{(k)(-)}^{t}(\omega)-\mathbf{p}_{(k)(-)}^{\prime t}(\omega)+\frac{1}{2 k_{(-)}(\omega)} \mathbf{I}_{(k)-}^{(n)}\left(\omega, \mathbf{g}^{t}\right)
\end{aligned}
$$

These functions, being analytic in $\mathbf{C}^{-}$for the first relations of (8.13) and (8.14) as well as in $\mathbf{C}^{+}$for the second relations of the same (8.13) and (8.14) and, moreover, being zero at infinity, must be equal to zero, i.e. $\mathbf{N}_{\alpha}(\omega)=\mathbf{0}$ and $\mathbf{N}_{k}(\omega)=\mathbf{0}$. 
Therefore, it follows that

$$
\begin{aligned}
& \mathbf{p}_{(\alpha)(+)}^{t}(\omega)=\mathbf{p}_{(\alpha)(+)}^{\prime t}(\omega), \quad \mathbf{p}_{(\alpha)(-)}^{t}(\omega)=\mathbf{p}_{(\alpha)(-)}^{\prime t}(\omega)-\frac{1}{2 \alpha_{(-)}(\omega)} \mathbf{I}_{(\alpha)-}^{(n)}\left(\omega, \mathbf{E}^{t}\right), \\
& \mathbf{p}_{(k)(+)}^{t}(\omega)=\mathbf{p}_{(k)(+)}^{\prime t}(\omega), \quad \mathbf{p}_{(k)(-)}^{t}(\omega)=\mathbf{p}_{(k)(-)}^{\prime t}(\omega)-\frac{1}{2 k_{(-)}(\omega)} \mathbf{I}_{(k)-}^{(n)}\left(\omega, \mathbf{g}^{t}\right) .
\end{aligned}
$$

If we introduce $\mathbf{E}_{n}^{t}(s)=\mathbf{E}^{t}(-s) \forall s \leq 0$ with its extension $\mathbf{E}_{n}^{t}(s)=\mathbf{0} \forall s>0$ and, analogously, $\mathbf{g}_{n}^{t}(s)=\mathbf{g}^{t}(-s) \forall s \leq 0$ with $\mathbf{g}_{n}^{t}(s)=\mathbf{0} \forall s>0$, whose Fourier transforms are

$$
\mathbf{E}_{n_{F}}^{t}(\omega)=\mathbf{E}_{n_{-}}^{t}(\omega)=\left(\mathbf{E}_{+}^{t}(\omega)\right)^{*}, \quad \mathbf{g}_{n_{F}}^{t}(\omega)=\mathbf{g}_{n_{-}}^{t}(\omega)=\left(\mathbf{g}_{+}^{t}(\omega)\right)^{*},
$$

then (8.5) and (8.6) assume the form

$$
\begin{aligned}
& \mathbf{I}_{(\alpha)}^{(\mathbf{R})}\left(\tau, \mathbf{E}^{t}\right)=-\int_{-\infty}^{+\infty} \alpha^{(e)}(\tau-s) \mathbf{E}_{n}^{t}(s) d s \\
& \mathbf{I}_{(k)}^{(\mathbf{R})}\left(\tau, \mathbf{g}^{t}\right)=-\int_{-\infty}^{+\infty} k^{(e)}(\tau-s) \mathbf{g}_{n}^{t}(s) d s
\end{aligned}
$$

so that

$$
\begin{aligned}
& \mathbf{I}_{(\alpha)_{F}}^{(\mathbf{R})}\left(\omega, \mathbf{E}^{t}\right)=-2 \alpha_{c}(\omega)\left(\mathbf{E}_{+}^{t}(\omega)\right)^{*} \\
& \mathbf{I}_{(k)_{F}}^{(\mathbf{R})}\left(\omega, \mathbf{g}^{t}\right)=-2 k_{c}(\omega)\left(\mathbf{g}_{+}^{t}(\omega)\right)^{*} .
\end{aligned}
$$

Thus, from (8.20) and (8.21), due to (7.13), we get

$$
\begin{gathered}
\frac{1}{2 \alpha_{(-)}(\omega)} \mathbf{I}_{(\alpha) F}^{(\mathbf{R})}\left(\omega, \mathbf{E}^{t}\right)=-\alpha_{(+)}(\omega)\left(\mathbf{E}_{+}^{t}(\omega)\right)^{*}, \\
\frac{1}{2 k_{(-)}(\omega)} \mathbf{I}_{(k)_{F}}^{(\mathbf{R})}\left(\omega, \mathbf{g}^{t}\right)=-k_{(+)}(\omega)\left(\mathbf{g}_{+}^{t}(\omega)\right)^{*} .
\end{gathered}
$$

On the other hand, using $(8.15)_{1}$ and $(8.16)_{1}$, the relations, analogous to (7.17) and $(7.18)$, written for $\mathbf{p}_{(\alpha)(+)}^{\prime t}(\omega)$ and $\mathbf{p}_{(k)(+)}^{\prime t}(\omega)$ taking into account (7.19) and (7.20) with (8.22) and (8.23), give

$$
\begin{aligned}
& \mathbf{p}_{(\alpha)(+)}^{t}(\omega)=\lim _{z \rightarrow \omega^{-}} \mathbf{p}_{(\alpha)(+)}^{\prime t}(z)=-\lim _{z \rightarrow \omega^{-}} \frac{1}{2 \pi i} \int_{-\infty}^{+\infty} \frac{\alpha_{(+)}\left(\omega^{\prime}\right)\left(\mathbf{E}_{+}^{t}\left(\omega^{\prime}\right)\right)^{*}}{\omega^{\prime}-z} d \omega^{\prime} \\
& \mathbf{p}_{(k)(+)}^{t}(\omega)=\lim _{z \rightarrow \omega^{-}} \mathbf{p}_{(k)(+)}^{\prime t}(z)=-\lim _{z \rightarrow \omega^{-}} \frac{1}{2 \pi i} \int_{-\infty}^{+\infty} \frac{k_{(+)}\left(\omega^{\prime}\right)\left(\mathbf{g}_{+}^{t}\left(\omega^{\prime}\right)\right)^{*}}{\omega^{\prime}-z} d \omega^{\prime}
\end{aligned}
$$

and hence

$$
\begin{aligned}
& \left(\mathbf{p}_{(\alpha)(+)}^{t}(\omega)\right)^{*}=\lim _{\xi \rightarrow \omega^{+}} \frac{1}{2 \pi i} \int_{-\infty}^{+\infty} \frac{\alpha_{(-)}\left(\omega^{\prime}\right) \mathbf{E}_{+}^{t}\left(\omega^{\prime}\right)}{\omega^{\prime}-\xi} d \omega^{\prime} \\
& \left(\mathbf{p}_{(k)(+)}^{t}(\omega)\right)^{*}=\lim _{\xi \rightarrow \omega^{+}} \frac{1}{2 \pi i} \int_{-\infty}^{+\infty} \frac{k_{(-)}\left(\omega^{\prime}\right) \mathbf{g}_{+}^{t}\left(\omega^{\prime}\right)}{\omega^{\prime}-\xi} d \omega^{\prime}
\end{aligned}
$$

Using the Plemelj formulae, we obtain

$$
\begin{aligned}
\alpha_{(-)}(\omega) \mathbf{E}_{+}^{t}(\omega) & =\mathbf{q}_{(\alpha)(-)}^{t}(\omega)-\mathbf{q}_{(\alpha)(+)}^{t}(\omega), \\
k_{(-)}(\omega) \mathbf{g}_{+}^{t}(\omega) & =\mathbf{q}_{(k)(-)}^{t}(\omega)-\mathbf{q}_{(k)(+)}^{t}(\omega),
\end{aligned}
$$


where

$$
\begin{aligned}
& \mathbf{q}_{(\alpha)( \pm)}^{t}(\omega)=\lim _{z \rightarrow \omega \mp} \frac{1}{2 \pi i} \int_{-\infty}^{+\infty} \frac{\alpha_{(-)}\left(\omega^{\prime}\right) \mathbf{E}_{+}^{t}\left(\omega^{\prime}\right)}{\omega^{\prime}-z} d \omega^{\prime}, \\
& \mathbf{q}_{(k)( \pm)}^{t}(\omega)=\lim _{z \rightarrow \omega \mp} \frac{1}{2 \pi i} \int_{-\infty}^{+\infty} \frac{k_{(-)}\left(\omega^{\prime}\right) \mathbf{g}_{+}^{t}\left(\omega^{\prime}\right)}{\omega^{\prime}-z} d \omega^{\prime} .
\end{aligned}
$$

We observe that the notation we have used here coincides with that of [3, 5]; so $\mathbf{q}_{(\alpha)}^{t}$ and $\mathbf{q}_{(k)}^{t}$ must not be confused with the heat flux.

Therefore, we have

$$
\left(\mathbf{p}_{(\alpha)(+)}^{t}(\omega)\right)^{*}=\mathbf{q}_{(\alpha)(-)}^{t}(\omega), \quad\left(\mathbf{p}_{(k)(+)}^{t}(\omega)\right)^{*}=\mathbf{q}_{(k)(-)}^{t}(\omega),
$$

and (7.26) becomes

$$
\begin{aligned}
\psi_{m}(t) & =\frac{1}{2}\left\{\frac{c}{\Theta_{0}} \vartheta^{2}(t)+\frac{1}{\varepsilon}[\varepsilon \mathbf{E}(t)+\vartheta(t) \mathbf{a}]^{2}+\mu \mathbf{H}^{2}(t)\right\} \\
& +\frac{1}{2 \pi} \int_{-\infty}^{+\infty}\left[\left|\mathbf{q}_{(\alpha)(-)}^{t}(\omega)\right|^{2}+\frac{1}{\Theta_{0}}\left|\mathbf{q}_{(k)(-)}^{t}(\omega)\right|^{2}\right] d \omega,
\end{aligned}
$$

which is the new expression of the minimum free energy in terms of $\mathbf{q}_{(\alpha)(-)}^{t}(\omega)$ and $\mathbf{q}_{(k)(-)}^{t}(\omega)$.

9. A particular model. We now consider the results obtained in the previous sections for a particular class of relaxation functions, which are characterized by two linear combinations of decaying exponentials, that is, we give explicit formulae for a discrete spectrum model material response.

Let

$$
\alpha(t)=\sum_{i=1}^{n} g_{i} e^{-\alpha_{i} t}, \quad k(t)=\sum_{i=1}^{n} h_{i} e^{-k_{i} t}, \quad n \in \mathbf{N},
$$

where the inverse decay times $\alpha_{i}, k_{i} \in \mathbf{R}^{++}(i=1,2, \ldots, n)$ and the coefficients $g_{i}$ and $h_{i}(i=1,2, \ldots, n)$ are assumed to be positive, too. We also put $\alpha_{1}<\alpha_{2}<\ldots<\alpha_{n}$ and $k_{1}<k_{2}<\ldots<k_{n}$.

From (9.1) we have, in particular,

$$
\alpha(0)=\sum_{i=1}^{n} g_{i}>0, \quad k(0)=\sum_{i=1}^{n} h_{i}>0,
$$

hence $(3.12)_{2}$ and the analogous relation for $\alpha$ [14 are satisfied. Then, we have the Fourier transforms

$$
\alpha_{F}(\omega)=\sum_{i=1}^{n} \frac{g_{i}}{\alpha_{i}+i \omega}, \quad k_{F}(\omega)=\sum_{i=1}^{n} \frac{h_{i}}{k_{i}+i \omega}, \quad \omega \in \mathbf{R},
$$

and hence we get, in particular,

$$
\alpha_{c}(\omega)=\sum_{i=1}^{n} \frac{\alpha_{i} g_{i}}{\alpha_{i}^{2}+\omega^{2}}, \quad k_{c}(\omega)=\sum_{i=1}^{n} \frac{k_{i} h_{i}}{k_{i}^{2}+\omega^{2}}, \quad \omega \in \mathbf{R} .
$$


Then, from (7.11) it follows that

$$
K^{(\alpha)}(\omega)=\sum_{i=1}^{n} \alpha_{i} g_{i} \frac{1+\omega^{2}}{\alpha_{i}^{2}+\omega^{2}}, \quad K^{(k)}(\omega)=\sum_{i=1}^{n} k_{i} h_{i} \frac{1+\omega^{2}}{k_{i}^{2}+\omega^{2}}, \quad \omega \in \mathbf{R},
$$

hence we get

$$
K_{\infty}^{(\alpha)}=\lim _{\omega \rightarrow \pm \infty} K^{(\alpha)}(\omega)=\sum_{i=1}^{n} \alpha_{i} g_{i}>0, \quad K_{\infty}^{(k)}=\lim _{\omega \rightarrow \pm \infty} K^{(k)}(\omega)=\sum_{i=1}^{n} k_{i} h_{i}>0 .
$$

The two functions $f^{(\alpha)}(z)=K^{(\alpha)}(\omega)$ and $f^{(k)}(z)=K^{(k)}(\omega)$ with $z=-\omega^{2}$ have a zero equal to one, which will be denoted by $\gamma_{1}^{2}=1$ and by $\delta_{1}^{2}=1$, respectively. If we consider the case when $1<\alpha_{1}^{2}$ and $1<k_{1}^{2}$ or the other one when $\alpha_{n}^{2}<1$ and $k_{n}^{2}<1$, it follows that $\alpha_{i}^{2} \neq 1$ and $k_{i}^{2} \neq 1(i=1,2, \ldots, n)$. In these cases the two functions have $n$ simple poles at $\alpha_{i}^{2}$ and $k_{i}^{2}(i=1,2, \ldots, n)$ and $n$ simple zeros denoted by $\gamma_{1}^{2}=1, \gamma_{j}^{2}$ $(j=2,3, \ldots, n)$ and $\delta_{1}^{2}=1, \delta_{j}^{2}(j=2,3, \ldots, n)$.

Only the conditions $\alpha_{i}^{2} \neq 1$ and $k_{i}^{2} \neq 1(i=1,2, \ldots, n)$ yield the possibility that $\alpha_{p}^{2}<1<\alpha_{p+1}^{2}$ and $k_{p^{\prime}}^{2}<1<k_{p^{\prime}+1}^{2}$, with $p$ equal to only one of the values $\{1,2, \ldots, n-1\}$ as well as $p^{\prime}$. In this case we have $\gamma_{1}^{2}=1_{\geq}^{<} \gamma_{p+1}^{2}$ and $\delta_{1}^{2}=1<\delta_{p^{\prime}+1}^{2}$ and it may occur that they coincide with 1 , which, therefore, has multiplicity 2 ; thus, the two functions have $n$ simple poles but only $n-1$ distinct zeros.

In all these cases the zeros $\gamma_{j}^{2}$ and $\delta_{j}^{2}(j=2,3, \ldots, n)$, i.e. those different from 1 , are such that

$$
\begin{aligned}
& \alpha_{1}^{2}<\gamma_{2}^{2}<\alpha_{2}^{2}<\ldots<\alpha_{p}^{2}<\gamma_{p+1}^{2}<\alpha_{p+1}^{2}<\ldots<\alpha_{n-1}^{2}<\gamma_{n}^{2}<\alpha_{n}^{2}, \\
& k_{1}^{2}<\delta_{2}^{2}<k_{2}^{2}<\ldots<k_{p^{\prime}}^{2}<\delta_{p^{\prime}+1}^{2}<k_{p^{\prime}+1}^{2}<\ldots<k_{n-1}^{2}<\delta_{n}^{2}<k_{n}^{2} .
\end{aligned}
$$

Therefore, let us suppose that $\alpha_{i}^{2} \neq 1, k_{i}^{2} \neq 1(i=1,2, \ldots, n)$. We can write $(9.5)$ as follows:

$$
K^{(\alpha)}(\omega)=K_{\infty}^{(\alpha)} \prod_{i=1}^{n}\left\{\frac{\gamma_{i}^{2}+\omega^{2}}{\alpha_{i}^{2}+\omega^{2}}\right\}, \quad K^{(k)}(\omega)=K_{\infty}^{(k)} \prod_{i=1}^{n}\left\{\frac{\delta_{i}^{2}+\omega^{2}}{k_{i}^{2}+\omega^{2}}\right\},
$$

from which we have, in particular,

$$
K_{(-)}^{(\alpha)}(\omega)=k_{\infty}^{(\alpha)} \prod_{i=1}^{n}\left\{\frac{\omega+i \gamma_{i}}{\omega+i \alpha_{i}}\right\}, \quad K_{(-)}^{(k)}(\omega)=k_{\infty}^{(k)} \prod_{i=1}^{n}\left\{\frac{\omega+i \delta_{i}}{\omega+i k_{i}}\right\},
$$

where

$$
k_{\infty}^{(\alpha)}=\sqrt{K_{\infty}^{(\alpha)}}, \quad k_{\infty}^{(k)}=\sqrt{K_{\infty}^{(k)}},
$$

$\gamma_{1}=1, \delta_{1}=1$ and eventually only one of the other zeros, say $\gamma_{p+1}$ and $\delta_{p^{\prime}+1}$, can coincide with $\gamma_{1}$ and $\delta_{1}$, that is, they have multiplicity 2 .

Thus, (7.14) and (9.10), being $\gamma_{1}^{2}=\delta_{1}^{2}=1$, yield

$$
\alpha_{(-)}(\omega)=i k_{\infty}^{(\alpha)} \frac{\prod_{j=2}^{n}\left(\omega+i \gamma_{j}\right)}{\prod_{i=1}^{n}\left(\omega+i \alpha_{i}\right)}, \quad k_{(-)}(\omega)=i k_{\infty}^{(k)} \frac{\prod_{j=2}^{n}\left(\omega+i \delta_{j}\right)}{\prod_{i=1}^{n}\left(\omega+i k_{i}\right)},
$$


which can be put in the form

$$
\alpha_{(-)}(\omega)=i k_{\infty}^{(\alpha)} \sum_{r=1}^{n} \frac{U_{r}^{(\alpha)}}{\omega+i \alpha_{r}}, \quad k_{(-)}(\omega)=i k_{\infty}^{(k)} \sum_{r=1}^{n} \frac{U_{r}^{(k)}}{\omega+i k_{r}}
$$

with

$$
U_{r}^{(\alpha)}=\frac{\prod_{j=2}^{n}\left(\gamma_{j}-\alpha_{r}\right)}{\prod_{i=1, i \neq r}^{n}\left(\alpha_{i}-\alpha_{r}\right)}, \quad U_{r}^{(k)}=\frac{\prod_{j=2}^{n}\left(\delta_{j}-k_{r}\right)}{\prod_{i=1, i \neq r}^{n}\left(k_{i}-k_{r}\right)} \quad(r=1,2, \ldots, n) .
$$

When one of the inverse decay times $\alpha_{i}$ as well as of $k_{i}(i=1,2, \ldots, n)$ is equal to 1 , we can consider these three cases: $(i) \alpha_{1}^{2} \equiv \gamma_{1}^{2}=1, k_{1}^{2} \equiv \delta_{1}^{2}=1 ;$ (ii) $\alpha_{n}^{2} \equiv \gamma_{1}^{2}=1$, $k_{n}^{2} \equiv \delta_{1}^{2}=1 ;($ iii $) \alpha_{p}^{2} \equiv \gamma_{1}^{2}=1, k_{p^{\prime}}^{2} \equiv \delta_{1}^{2}=1$. In these cases we have different expressions for $K^{(\alpha)}(\omega)$ and $K^{(k)}(\omega)$, but the final expressions of $\alpha_{(-)}(\omega)$ and $k_{(-)}(\omega)$, given by (9.13), do not change, as well as the expressions of the coefficients $U_{r}^{(\alpha)}$ and $U_{r}^{(k)}$, given by (9.14). The only differences, with respect to the previous case with $\alpha_{i}^{2} \neq 1$ and $k_{i}^{2} \neq 1$, are the following ones: in (9.14) we must put $\alpha_{1}=1$ and $k_{1}=1$ in the case $(i), \alpha_{n}=1$ and $k_{n}=1$ in the case $(i i)$ and $\alpha_{p}=1$ and $k_{p^{\prime}}=1$ in the case (iii).

Therefore, we can use (9.13) and (9.14) in any case to obtain the expression of the minimum free energy. However, we observe that they are consistent only if $n>1$; thus, the case when $n=1$ must be studied directly from (9.10), where we have one zero both for $K^{(\alpha)}(\omega)$ and $K^{(k)}(\omega): \gamma_{1}=1, \delta_{1}=1$; the expressions (7.14) written for $n=1$ yield $U_{1}^{(\alpha)}=1, U_{1}^{(k)}=1$.

Thus, (8.30) and (8.31), taking account of (9.13), yield

$$
\begin{aligned}
& \mathbf{q}_{(\alpha)(-)}^{t}(\omega)=\frac{1}{2 \pi i} \sum_{r=1}^{n} i k_{\infty}^{(\alpha)} U_{r}^{(\alpha)} \int_{-\infty}^{+\infty} \frac{\mathbf{E}_{+}^{t}\left(\omega^{\prime}\right) /\left(\omega^{\prime}-\omega^{+}\right)}{\omega^{\prime}-\left(-i \alpha_{r}\right)} d \omega^{\prime}, \\
& \mathbf{q}_{(k)(-)}^{t}(\omega)=\frac{1}{2 \pi i} \sum_{r=1}^{n} i k_{\infty}^{(k)} U_{r}^{(k)} \int_{-\infty}^{+\infty} \frac{\mathbf{g}_{+}^{t}\left(\omega^{\prime}\right) /\left(\omega^{\prime}-\omega^{+}\right)}{\omega^{\prime}-\left(-i k_{r}\right)} d \omega^{\prime} .
\end{aligned}
$$

These quantities can be evaluated by closing on $\mathbf{C}^{(-)}$. Taking account of the sense of the integrations, we obtain

$$
\begin{aligned}
& \mathbf{q}_{(\alpha)(-)}^{t}(\omega)=i k_{\infty}^{(\alpha)} \sum_{r=1}^{n} \frac{U_{r}^{(\alpha)}}{\omega+i \alpha_{r}} \mathbf{E}_{+}^{t}\left(-i \alpha_{r}\right), \\
& \mathbf{q}_{(k)(-)}^{t}(\omega)=i k_{\infty}^{(k)} \sum_{r=1}^{n} \frac{U_{r}^{(k)}}{\omega+i k_{r}} \mathbf{g}_{+}^{t}\left(-i k_{r}\right),
\end{aligned}
$$

so that

$$
\begin{aligned}
& \left(\mathbf{q}_{(\alpha)(-)}^{t}(\omega)\right)^{*}=-i k_{\infty}^{(\alpha)} \sum_{r=1}^{n} \frac{U_{r}^{(\alpha)}}{\omega-i \alpha_{r}}\left(\mathbf{E}_{+}^{t}\left(-i \alpha_{r}\right)\right)^{*} \\
& \left(\mathbf{q}_{(k)(-)}^{t}(\omega)\right)^{*}=-i k_{\infty}^{(k)} \sum_{r=1}^{n} \frac{U_{r}^{(k)}}{\omega-i k_{r}}\left(\mathbf{g}_{+}^{t}\left(-i \alpha_{r}\right)\right)^{*}
\end{aligned}
$$


where, by virtue of $(3.6)_{1}$,

$$
\begin{gathered}
\mathbf{E}_{+}^{t}\left(-i \alpha_{r}\right)=\int_{0}^{+\infty} \mathbf{E}^{t}(s) e^{-\alpha_{r} s} d s=\left(\mathbf{E}_{+}^{t}\left(-i \alpha_{r}\right)\right)^{*}, \\
\mathbf{g}_{+}^{t}\left(-i k_{r}\right)=\int_{0}^{+\infty} \mathbf{g}^{t}(s) e^{-k_{r} s} d s=\left(\mathbf{g}_{+}^{t}\left(-i k_{r}\right)\right)^{*}
\end{gathered}
$$

Thus, using (9.17)-(9.22), we get

$$
\begin{aligned}
\frac{1}{2 \pi} \int_{-\infty}^{+\infty} & \left|\mathbf{q}_{(\alpha)(-)}^{t}(\omega)\right|^{2} d \omega \\
& =K_{\infty}^{(\alpha)} \sum_{r, l=1}^{n} U_{r}^{(\alpha)} U_{l}^{(\alpha)} \mathbf{E}_{+}^{t}\left(-i \alpha_{r}\right) \cdot \mathbf{E}_{+}^{t}\left(-i \alpha_{l}\right) \frac{1}{2 \pi i} \int_{-\infty}^{+\infty} \frac{i /\left(\omega+i \alpha_{r}\right)}{\omega-i \alpha_{l}} d \omega \\
& =K_{\infty}^{(\alpha)} \sum_{r, l=1}^{n} \frac{U_{r}^{(\alpha)} U_{l}^{(\alpha)}}{\alpha_{r}+\alpha_{l}} \mathbf{E}_{+}^{t}\left(-i \alpha_{r}\right) \cdot \mathbf{E}_{+}^{t}\left(-i \alpha_{l}\right)
\end{aligned}
$$

and

$$
\begin{aligned}
\frac{1}{2 \pi} \int_{-\infty}^{+\infty} \mid & \left.\mathbf{q}_{(k)(-)}^{t}(\omega)\right|^{2} d \omega \\
& =K_{\infty}^{(k)} \sum_{r, l=1}^{n} U_{r}^{(k)} U_{l}^{(k)} \mathbf{g}_{+}^{t}\left(-i k_{r}\right) \cdot \mathbf{g}_{+}^{t}\left(-i k_{l}\right) \frac{1}{2 \pi i} \int_{-\infty}^{+\infty} \frac{i /\left(\omega+i k_{r}\right)}{\omega-i k_{l}} d \omega \\
& =K_{\infty}^{(k)} \sum_{r, l=1}^{n} \frac{U_{r}^{(k)} U_{l}^{(k)}}{k_{r}+k_{l}} \mathbf{g}_{+}^{t}\left(-i k_{r}\right) \cdot \mathbf{g}_{+}^{t}\left(-i k_{l}\right),
\end{aligned}
$$

which allow us to obtain from (8.33), on account of $(9.21)_{1}$ and $(9.22)_{1}$,

$$
\begin{aligned}
\psi_{m}(t)=\frac{1}{2}\{ & \left.\frac{c}{\Theta_{0}} \vartheta^{2}(t)+\frac{1}{\varepsilon}[\varepsilon \mathbf{E}(t)+\vartheta(t) \mathbf{a}]^{2}+\mu \mathbf{H}^{2}(t)\right\} \\
& +\frac{1}{2} \int_{0}^{+\infty} \int_{0}^{+\infty} 2\left[\sum_{r, l=1}^{n} K_{\infty}^{(\alpha)} \frac{U_{r}^{(\alpha)} U_{l}^{(\alpha)}}{\alpha_{r}+\alpha_{l}} e^{-\alpha_{r} s_{1}-\alpha_{l} s_{2}} \mathbf{E}^{t}\left(s_{1}\right) \cdot \mathbf{E}^{t}\left(s_{2}\right)\right. \\
& \left.+\frac{1}{\Theta_{0}} \sum_{r, l=1}^{n} K_{\infty}^{(k)} \frac{U_{r}^{(k)} U_{l}^{(k)}}{k_{r}+k_{l}} e^{-k_{r} s_{1}-k_{l} s_{2}} \mathbf{g}^{t}\left(s_{1}\right) \cdot \mathbf{g}^{t}\left(s_{2}\right)\right] d s_{1} d s_{2} .
\end{aligned}
$$

In the particular case when $n=1$ the minimum free energy has a meaningful form. It follows from (9.25), taking into account that $U_{1}^{(\alpha)}=1$ and $U_{1}^{(k)}=1$, as we have already observed, and that from (9.6) we have

$$
K_{\infty}^{(k)}=\alpha_{1} g_{1}, \quad K_{\infty}^{(k)}=k_{1} h_{1} ;
$$

we get

$$
\begin{aligned}
\psi_{m}(t)= & \frac{1}{2}\left\{\frac{c}{\Theta_{0}} \vartheta^{2}(t)+\frac{1}{\varepsilon}[\varepsilon \mathbf{E}(t)+\vartheta(t) \mathbf{a}]^{2}+\mu \mathbf{H}^{2}(t)\right\} \\
& +\frac{1}{2}\left\{g_{1}\left[\int_{0}^{+\infty} \mathbf{E}^{t}(s) e^{-\alpha_{1} s} d s\right]^{2}+\frac{1}{\Theta_{0}} h_{1}\left[\int_{0}^{+\infty} \mathbf{g}^{t}(s) e^{-k_{1} s} d s\right]^{2}\right\} .
\end{aligned}
$$




\section{REFERENCES}

[1] W.A. Day, Reversibility, recoverable work and free energy in linear viscoelasticity, Quart. J. Mech. Appl. Math. 23, 1-15 (1970)

[2] S. Breuer and E.T. Onat, On recoverable work in viscoelasticity, Z. Angew. Math. Phys. 15, 13-21 (1981) MR0178644(31:2901)

[3] J.M. Golden, Free energy in the frequency domain: the scalar case, Quart. Appl. Math. LVIII (1), 127-150 (2000) MR.1739041 (2001e:74025)

[4] G. Gentili, Thermodynamic potentials for electromagnetic fields in the ionosphere, Int. J. Engng. Sci. (33) 11, 1561-1575 (1995) MR1343117 (96h:78001)

[5] G. Gentili, Maximum recoverable work, minimum free energy and state space in linear viscoelasticity, Quart. Appl. Math. LX (1), 153-182 (2002) MR.1878264 (2002m:74011)

[6] G. Gentili and V. Berti, The minimum free energy for isothermal dielectrics with memory, J. Non-equil. Thermodyn. 24, 154-176 (1999)

[7] M. Fabrizio, Teoremi di approssimazione e restrizioni termodinamiche per le equazioni costitutive del campo elettromagnetico, Atti Accad. Sci. Ist. Bologna XII (10), 97 (1973) MR0347238(49:11958)

[8] M. Fabrizio and J.M. Golden, Maximum and minimum free energies for a linear viscoelastic material, Quart. Appl. Math. LX (2), 341-381 (2002) MR.1900497(2003b:74013)

[9] M. Fabrizio and A. Morro, Mathematical problems in linear viscoelasticity, SIAM Studies in Applied Mathematics, Philadelphia, 1992 MR.1153021 (93a:73034)

[10] M. Fabrizio and A. Morro, Thermodynamics of electromagnetic isothermal systems with memory, J. Non-equil. Thermodyn. 22, 110-128 (1997)

[11] M. Fabrizio and A. Morro, Electromagnetism of continuous media, Oxford University Press, 2003 MR.1996323 (2004j:78001)

[12] M. Fabrizio, C. Giorgi and A. Morro, Free energies and dissipation properties for systems with memory, Arch. Rational Mech. Anal. 125, 341-373 (1994) MR 1253168 (95j:73012)

[13] G. Amendola, Asymptotic behaviour for electromagnetic fields in the ionosphere, Ann. Univ. Ferrara Sez. VII - Sci. Mat. XLVIII, 165-187 (2002) MR1980831 (2004f:86006)

[14] G. Amendola, The minimum free energy for an electromagnetic conductor, Appl. Anal. (1) 84, 67-87 (2005) MR2113656

[15] G. Amendola, Linear stability for a thermoelectromagnetic material with memory, Quart. Appl. Math. LIX (1), 67-84 (2002) MR1811095 (2001j:35261)

[16] G. Del Piero and L. Deseri, On the concepts of state and free energy in linear viscoelasticity, Arch. Rational Mech. Anal. 138, 1-35 (1997) MR.1463802 (98i:73023)

[17] M.E. Gurtin and A.C. Pipkin, A general theory of heat conduction with finite wave speeds, Arch. Rational Mech. Anal. 31, 113-126 (1968)

[18] C. Cattaneo, Sulla conduzione del calore, Atti Sem. Mat. Fis. Univ. Modena 3, 83-101 (1948) MR0032898 (11:362d)

[19] B.D. Coleman and E.H. Dill, On the thermodynamics of electromagnetic fields in materials with memory, Arch. Rational Mech. Anal. 41, 132-162 (1971) MR0347245 (49:11965)

[20] B.D. Coleman and E.H. Dill, Thermodynamic restrictions on the constitutive equations of electromagnetic theory, ZAMP 22, 691-702 (1971)

[21] B.D. Coleman and D.R. Owen, A mathematical foundation of Thermodynamics, Arch. Rational Mech. Anal. 54, 1-104 (1974) MR0395502 (52:16299)

[22] W. Noll, A new mathematical theory of simple materials, Arch. Rational Mech. Anal. 48, 1-50 (1972) MR0445985 (56:4318)

[23] N.I. Muskhelishvili, Singular Integral Equations, Noordhoff, Groningen, 1953 MR0058845|(15:434e) 